\title{
On a Spectral Version of Cartan's Theorem
}

\author{
Sayani Bera ${ }^{1}\left(\mathbb{D} \cdot\right.$ Vikramjeet Singh Chandel $^{2} \cdot$ Mayuresh Londhe $^{3}$
}

Received: 27 June 2021 / Accepted: 20 July 2021 / Published online: 7 January 2022

(c) Mathematica Josephina, Inc. 2021

\begin{abstract}
For a domain $\Omega$ in the complex plane, we consider the domain $S_{n}(\Omega)$ consisting of those $n \times n$ complex matrices whose spectrum is contained in $\Omega$. Given a holomorphic self-map $\Psi$ of $S_{n}(\Omega)$ such that $\Psi(A)=A$ and the derivative of $\Psi$ at $A$ is identity for some $A \in S_{n}(\Omega)$, we investigate when the map $\Psi$ would be spectrum-preserving. We prove that if the matrix $A$ is either diagonalizable or non-derogatory then for most domains $\Omega, \Psi$ is spectrum-preserving on $S_{n}(\Omega)$. Further, when $A$ is arbitrary, we prove that $\Psi$ is spectrum-preserving on a certain analytic subset of $S_{n}(\Omega)$.
\end{abstract}

Keywords Spectrum-preserving maps $\cdot$ Symmetrized product $\cdot$ Iteration theory

Mathematics Subject Classification Primary: 32H02 - 32H50; Secondary: 47A56 . 32F45

\section{Introduction and Statement of Main Results}

A well-known result of Cartan about holomorphic self-maps, also known as Cartan's uniqueness theorem, says: every holomorphic self-map of a bounded domain (in the complex Euclidean space) that has a fixed point so that the derivative of the holomorphic map at the fixed point is identity has to be the identity map on the given bounded domain. The above result was generalized to taut complex manifolds by Wu [21] and shortly later to the case of Kobayashi hyperbolic complex manifolds by

\footnotetext{
Sayani Bera

sayanibera2016@gmail.com

Vikramjeet Singh Chandel

abelvikram@gmail.com ; vikramjeetchandel@hri.res.in

Mayuresh Londhe

mayureshl@iisc.ac.in

1 Indian Association for the Cultivation of Science, Kolkata 700032, India

2 Harish-Chandra Research Institute, Prayagraj (Allahabad) 211019, India

3 Department of Mathematics, Indian Institute of Science, Bangalore 560012, India
} 
Kobayashi [13]. The purpose of this article is to explore holomorphic self-maps of certain matricial domains - that are not Kobayashi hyperbolic (thus not taut) - in the spirit of Cartan's Theorem. We begin with introducing these domains.

Given $n \in \mathbb{N}, n \geq 2$, we denote by $M_{n}(\mathbb{C})$ the set of all $n \times n$ complex matrices. For a matrix $W \in M_{n}(\mathbb{C})$, the spectrum of $W$ is the set of eigenvalues of $W$ and is denoted by $\sigma(W)$. Let $\Omega$ be a domain in the complex plane $\mathbb{C}$, we consider the set

$$
S_{n}(\Omega):=\left\{W \in M_{n}(\mathbb{C}): \sigma(W) \subset \Omega\right\}
$$

Note that $S_{n}(\Omega)$ is an open and connected subset of $M_{n}(\mathbb{C}) \equiv \mathbb{C}^{n^{2}}$. In the case when $\Omega=\mathbb{D}$, where $\mathbb{D}$ is the open unit disc in $\mathbb{C}$ centred at the origin, the domain $S_{n}(\mathbb{D})$ is called the spectral unit ball. In [17], Ransford-White initiated function-theoretic study of the spectral unit ball. Since then, the spectral unit ball has been studied intensively in the literature, see, for instance $[3,7,10,16,22]$ and the references therein. We now enlist an important observation about the domains $S_{n}(\Omega)$.

Lemma 1.1 For any domain $\Omega \subset \mathbb{C}$ and $n \geq 2$, the domain $S_{n}(\Omega)$ is not Kobayashi hyperbolic.

In fact, for every $S_{n}(\Omega), n \geq 2$, and for any $W \in S_{n}(\Omega)$ there exists a non-constant holomorphic map $f_{W}: \mathbb{C} \longrightarrow S_{n}(\Omega)$ such that $\sigma(f(\cdot))=\sigma(W)$ on $\mathbb{C}$. We postpone the proof of Lemma 1.1 to Sect. 2, where we also recall several relevant definitions and results. Thus given a holomorphic self-map $\Psi$ of $S_{n}(\Omega), n \geq 2$, such that $\Psi(A)=A$ and the derivative of $\Psi$ at $A$ is identity, i.e. $\Psi^{\prime}(A)=\mathbb{I}$, the aforementioned results of Kobayashi and Wu cannot be directly applied to conclude that $\Psi$ is the identity map on $S_{n}(\Omega)$. Indeed, there exists a holomorphic self-map $\Psi$ of $S_{2}(\mathbb{D})$ such that $\Psi(0)=0$ and $\Psi^{\prime}(0)=\mathbb{I}$ that is not even injective (see [17, Sect. 0]).

To study the holomorphic self-maps of $S_{n}(\Omega)$, we employ its relation with the $n$-th symmetrized product of $\Omega$ which, in general, have many nice properties. One such important property is that-while none of the domains $S_{n}(\Omega)$ are Kobayashi hyperbolic - the $n$-th symmetrized product of $\Omega$ is Kobayashi hyperbolic for most domains $\Omega \subset \mathbb{C}$. To define this latter object, we consider the symmetrization map $\pi_{n}: \mathbb{C}^{n} \longrightarrow \mathbb{C}^{n}$ defined by $\pi_{n}(z):=\left(\pi_{n, 1}(z), \ldots, \pi_{n, j}(z), \ldots, \pi_{n, n}(z)\right)$, where $\pi_{n, j}(z)$ is the $j$ th elementary symmetric polynomial in variables $z_{1}, \ldots, z_{n}$ for $z:=$ $\left(z_{1}, \ldots, z_{n}\right)$. In other words, we have

$$
\prod_{j=1}^{n}\left(t-z_{j}\right)=t^{n}+\sum_{j=1}^{n}(-1)^{j} \pi_{n, j}\left(z_{1}, \ldots, z_{n}\right) t^{n-j}, \quad t \in \mathbb{C} .
$$

The $n$-th symmetrized product of $\Omega$, denoted by $\Sigma^{n}(\Omega)$, is defined by $\Sigma^{n}(\Omega):=$ $\pi_{n}\left(\Omega^{n}\right)$. Since the symmetrization map $\pi_{n}: \mathbb{C}^{n} \longrightarrow \mathbb{C}^{n}$ is a proper holomorphic map, it follows that $\Sigma^{n}(\Omega)$ is a domain in $\mathbb{C}^{n}$. 
The aforementioned relation between $S_{n}(\Omega)$ and $\Sigma^{n}(\Omega)$ is via the map c : $M_{n}(\mathbb{C}) \longrightarrow \mathbb{C}^{n}$ defined by $\mathbf{c}(W):=\left(c_{1}(W), \ldots, c_{n}(W)\right)$ where the polynomial

$$
t^{n}+\sum_{k=1}^{n}(-1)^{k} c_{k}(W) t^{n-k}
$$

is the characteristic polynomial of $W$. We shall denote the restriction of the map $\mathbf{c}$ to any open subset of $M_{n}(\mathbb{C})$ by $\mathbf{c}$ itself. Observe for each $k, 1 \leq k \leq n$, since $c_{k}(W)$ is the sum of all principal minors of order $k$ of the matrix $W$, $\mathbf{c}$ is a holomorphic map on $M_{n}(\mathbb{C})$. Further, if $\left\{\lambda_{1}, \ldots, \lambda_{n}\right\}$ is a list of eigenvalues of $W$, repeated with algebraic multiplicity, then $c_{k}(W)=\pi_{n, k}\left(\lambda_{1}, \ldots, \lambda_{n}\right)$ for each $k$, where $c_{k}(W)$ is the $k$ th coordinate of $\mathbf{c}(W)$. It now follows that

$$
\mathbf{c}\left(S_{n}(\Omega)\right)=\Sigma^{n}(\Omega) \text { and } S_{n}(\Omega)=\mathbf{c}^{-1}\left(\Sigma^{n}(\Omega)\right)
$$

for any domain $\Omega \subset \mathbb{C}$. It turns out that the domain $\Sigma^{n}(\Omega)$ is Kobayashi hyperbolic (and also Kobayashi complete) if and only if the cardinality of $\mathbb{C} \backslash \Omega$ is at least $2 n$ (see Result 3.1). In Sect. 3, using the Kobayashi hyperbolicity of $\Sigma^{n}(\Omega)$, we prove that every holomorphic self-map $\Psi$ of $S_{n}(\Omega)$ induces a unique holomorphic self-map $G_{\Psi}$ of $\Sigma^{n}(\Omega)$ such that $\mathbf{c} \circ \Psi=G_{\Psi} \circ \mathbf{c}$, i.e. the following diagram commutes:

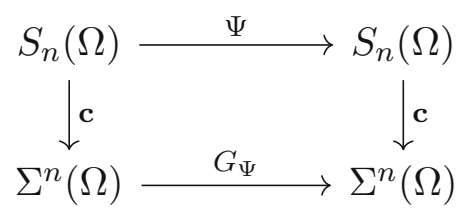

Fig. 1 Existence of $G_{\Psi}$

By studying the map $G_{\Psi}$, we are led to the first main result of this article.

Theorem 1.2 Given $n \in \mathbb{N}, n \geq 2$, and a domain $\Omega \subset \mathbb{C}$ satisfying $\#(\mathbb{C} \backslash \Omega) \geq 2 n$. Let $\Psi$ be a holomorphic self-map of $S_{n}(\Omega)$ such that $\Psi(A)=A$ and $\Psi^{\prime}(A)=\mathbb{I}$ for some $A \in S_{n}(\Omega)$. Assume that the matrix $A$ is either a diagonalizable matrix or a non-derogatory matrix. Then

$$
\mathbf{c}(\Psi(W))=\mathbf{c}(W) \text { for every } W \in S_{n}(\Omega) .
$$

Consequently, $\sigma(\Psi(W))=\sigma(W)$ and the algebraic multiplicity of each eigenvalue is preserved for every $W \in S_{n}(\Omega)$, i.e. $\Psi$ is spectrum-preserving on $S_{n}(\Omega)$.

Remark 1.3 Recall that a non-derogatory matrix is a matrix for which the characteristic polynomial and the minimal polynomial are same, see [11, p. 195] for other equivalent definitions. Observe that the set of diagonalizable matrices is dense in $S_{n}(\Omega)$, and the set of non-derogatory matrices is open and dense in $S_{n}(\Omega)$ for any domain $\Omega$ in $\mathbb{C}$. 
Furthermore, given any $A \in S_{2}(\Omega)$, it is either a diagonalizable matrix or a nonderogatory matrix. Hence, when $n=2$, the condition on the matrix $A$ in Theorem 1.2 is superfluous.

As an application of Theorem 1.2, we prove a result that gives stronger conclusion in a neighbourhood of the matrix $A$ than that of Theorem 1.2.

Corollary 1.4 Let $n, \Omega, \Psi$ and $A$ be as in Theorem 1.2. Then there exists a neighbourhood $\mathcal{N}$ of $A$ such that $\Psi(W)$ is conjugate to $W$ for any $W \in \mathcal{N}$.

Remark 1.5 Note that without any condition on $\Omega$ in Theorem 1.2, $\Psi$ need not be spectrum-preserving. For example: let $\Omega=\mathbb{C} \backslash\{0\}$ and consider $\Psi(W):=\exp (W-\mathbb{I})$. Notice that $\Psi$ satisfies $\Psi(\mathbb{I})=\mathbb{I}$ and $\Psi^{\prime}(\mathbb{I})=\mathbb{I}$ but $\Psi$ is not spectrum-preserving. When $\Omega=\mathbb{D}$ and $A=0 \in S_{n}(\mathbb{D}), n \geq 2$, the above theorem was proved by Ransford-White [17, Theorem 3]. Since the automorphism group of $S_{n}(\mathbb{D})$ is far from being transitive (see [17, Theorem 4]), one cannot use the result due to RansfordWhite to deduce the conclusion of the above theorem for $S_{n}(\mathbb{D})$ and for an arbitrary $A \neq 0$.

We must mention that our proof of Theorem 1.2 is not a routine extension of the argument given by Ransford-White in the case mentioned above. We provide a very short sketch of the proof of Theorem 1.2 to point out some features of it that are novel. Let $\Psi$ be as in Theorem 1.2 and $G_{\Psi}$ be the self-map of $\Sigma^{n}(\Omega)$ associated with $\Psi$ (see Fig. 1). Observe that if we show that $G_{\Psi}$ is the identity map on $\Sigma^{n}(\Omega)$ then Theorem 1.2 follows. To study the set of fixed points of $G_{\Psi}$, we introduce the technique of local decomposition of the map c, which is particularly useful when the matrix $A$ is non-zero.

- Let $\lambda_{i}$ be an eigenvalue of $A$ with algebraic multiplicity $n_{i}, i \in\{1,2, \ldots, m\}$. Then the map $\mathbf{c}$ decomposes locally as $\mathbf{c}=\tau \circ \theta$, where $\theta$ is a map into the cartesian product of $\Sigma^{n_{i}}(\Omega)$, i.e. $\prod_{i=1}^{m} \Sigma^{n_{i}}(\Omega)$ and $\tau$ is the canonical map from $\prod_{i=1}^{m} \Sigma^{n_{i}}(\Omega)$ onto $\Sigma^{n}(\Omega)$. Furthermore, we observe that $\tau$ is a local biholomorphism. This allows us to define the holomorphic map $F_{\Psi}$-in a neighbourhood of $\theta(A)$-which is locally a biholomorphic conjugate of $G_{\Psi}$ via the map $\tau$ (see Fig. 2). Note this step is independent of the choice of the matrix $A$.

- When $A$ is diagonalizable, using a result on the perturbation of eigenvalues of a normal matrix by Sun [19], we prove that the trace of $F_{\Psi}^{\prime}$ at $\theta(A)$ is $n$, where $F_{\Psi}$ is as above. This implies that the trace of $G_{\Psi}^{\prime}$ at $\mathbf{c}(A)$ is $n$. On the other hand, when $A$ is non-derogatory, we explicitly construct a right inverse of the map c passing through the point $A$ which, in particular, shows that $G_{\Psi}^{\prime}(\mathbf{c}(A))=\mathbb{I}$.

- Under the cardinality condition $\#(\mathbb{C} \backslash \Omega) \geq 2 n$, the domain $\Sigma^{n}(\Omega)$ is Kobayashi complete. We appeal to results from the iteration theory of holomorphic self-maps on taut complex manifolds (see Sect. 2) - together with the information about $G_{\Psi}^{\prime}(\mathbf{c}(A))$ above- to establish that $G_{\Psi}$ is the identity map on $\Sigma^{n}(\Omega)$.

Remark 1.6 Sun's result-alluded to as above_-gives a bound on the eigenvalues for the perturbation of a normal matrix (see Result 4.3 for the statement). In general, similar bounds on eigenvalues for perturbation of an arbitrary matrix have robust 
error; see, for instance $[8,18]$. Further, given a matrix $B$ if there exists a local right inverse of the map c passing through the point $B$, then $B$ has to be a non-derogatory matrix. Therefore, the techniques used to prove Theorem 1.2 do not extend.

We now turn to the case when the matrix $A$ in Theorem 1.2 is not necessarily diagonalizable or non-derogatory. Notice, if we take derivatives on both sides of $\mathbf{c} \circ$ $\Psi=G_{\Psi} \circ \mathbf{c}$ at $A$ then it follows that the range space of the derivative of $\mathbf{c}$ at $A$ lies in the eigenspace of the derivative of $G_{\Psi}$ at $\mathbf{c}(A)$ corresponding to the eigenvalue 1 . This eigenspace plays an important role with regard to the spectrum-preserving property of $\Psi$. Now, the rank of $\mathbf{c}$ at $A$ gives a lower bound on the dimension of the eigenspace of $G_{\Psi}^{\prime}(\mathbf{c}(A))$ corresponding to the eigenvalue 1 . In this direction, we have the following proposition, which is interesting in its own right:

Proposition 1.7 Let $A \in M_{n}(\mathbb{C})$ be given. Then the rank of the derivative of $\mathbf{c}$ at $A$ is equal to the degree of the minimal polynomial of $A$.

Our proof of Proposition 1.7 crucially uses the local decomposition of $\mathbf{c}$ as described before. By a result of Vigué [20] (see Sect. 2) about the fixed-point set of holomorphic self-maps, the eigenspace of $G_{\Psi}^{\prime}(\mathbf{c}(A))$ corresponding to the eigenvalue 1 determines the fixed-point set of $G_{\Psi}$. Using Proposition 1.7-in a way that is described in the last paragraph — we get a lower bound on the dimension of the fixed-point set of $G_{\Psi}$ which leads to the second main result of this article.

Theorem 1.8 Given $n \in \mathbb{N}, n \geq 2$, and a domain $\Omega \subset \mathbb{C}$ satisfying $\#(\mathbb{C} \backslash \Omega) \geq 2 n$. Let $\Psi$ be a holomorphic self-map of $S_{n}(\Omega)$ such that $\Psi(A)=A$ and $\Psi^{\prime}(A)=\mathbb{I}$. Then there is a closed complex submanifold $\mathscr{S}$ of $\Sigma^{n}(\Omega)$ containing $\mathbf{c}(A)$ of complex dimension greater than or equal to the degree of the minimal polynomial of A such that for every $W \in \mathbf{c}^{-1}(\mathscr{S})$ we have $\mathbf{c}(\Psi(W))=\mathbf{c}(W)$.

Since for a non-derogatory matrix the degree of the minimal polynomial is maximal, Theorem 1.8 gives an alternate proof of Theorem 1.2 when $A$ is non-derogatory. We prove Theorems 1.2 and 1.8 in Sects. 5 and 7, respectively, while the proof of Proposition 1.7 is given in Sect. 6.

Concluding remarks For a domain $\Omega$ with $\#(\mathbb{C} \backslash \Omega) \geq 2 n$, the map $\Psi$ as in Theorem 1.2 is spectrum-preserving when the matrix $A$ belongs to a large subset of $S_{n}(\Omega)$ (see Remark 1.3). Thus it seems that the same conclusion should hold for any choice of $A$, but current tools and results are not enough to conclude this. For example, when $n=3$, there is one particular choice of the matrix $A$ for which we are not able to say whether $\Psi$ is spectrum-preserving (see Sect. 7). It would be interesting to find a counterexample in this case.

\section{Kobayashi Hyperbolicity and Iteration Theory on Taut Complex Manifolds}

In this section, we recall notions of Kobayashi hyperbolicity, Kobayashi completeness and tautness for a given complex manifold. As hinted in Sect. 1, we shall need results 
from the iteration theory of holomorphic self-maps on taut complex manifolds in our proofs, so we state those results too in this section. Before we begin, a piece of notation-given complex manifolds $\mathscr{X}$ and $\mathscr{Y}$, we shall denote by $\mathcal{O}(\mathscr{X}, \mathscr{Y})$ the set of all holomorphic maps from $\mathscr{X}$ into $\mathscr{Y}$.

Let $\mathscr{X}$ be a complex manifold and let $h$ denote the hyperbolic distance induced by the Poincaré metric on the unit disc $\mathbb{D}$. The Kobayashi pseudo-distance $K_{\mathscr{X}}$ : $\mathscr{X} \times \mathscr{X} \longrightarrow[0, \infty)$ is defined by: given two points $p, q \in \mathscr{X}$,

$$
K_{\mathscr{X}}(p, q):=\inf \left\{\sum_{i=1}^{k} h\left(\zeta_{i-1}, \zeta_{i}\right):\left(\phi_{1}, \ldots, \phi_{k} ; \zeta_{0}, \ldots, \zeta_{k}\right) \in \mathcal{A}(p, q)\right\}
$$

where $\mathcal{A}(p, q)$ is the set of all analytic chains in $\mathscr{X}$ joining $p$ to $q$. Here, $\left(\phi_{1}, \ldots, \phi_{k} ; \zeta_{0}, \ldots, \zeta_{k}\right)$ is an analytic chain in $\mathscr{X}$ joining $p$ to $q$ if $\phi_{i} \in \mathcal{O}(\mathbb{D}, \mathscr{X})$ for each $i$ such that

$$
p=\phi_{1}\left(\zeta_{0}\right), \quad \phi_{k}\left(\zeta_{k}\right)=q \text { and } \phi_{i}\left(\zeta_{i}\right)=\phi_{i+1}\left(\zeta_{i}\right)
$$

for $i=1, \ldots, k-1$.

It is not difficult to check that $K_{\mathscr{X}}$ is a pseudo-distance. Using the Schwarz lemma on the unit disc $\mathbb{D}$, we see that $K_{\mathbb{D}} \equiv h$. An important property of the Kobayashi pseudo-distance is its contractivity under holomorphic maps, i.e. if $F: \mathscr{X} \longrightarrow \mathscr{Y}$ is a holomorphic map then $K_{\mathscr{Y}}(F(p), F(q)) \leq K \mathscr{X}(p, q)$ for all $p, q \in \mathscr{X}$. A complex manifold $\mathscr{X}$ is called Kobayashi hyperbolic if the pseudo-distance $K_{\mathscr{X}}$ is a distance, i.e. $K_{\mathscr{X}}(p, q)=0$ if and only if $p=q$. Furthermore, $\mathscr{X}$ is called Kobayashi complete if it is Kobayashi hyperbolic and the metric space $(\mathscr{X}, K \mathscr{X})$ is complete. It is a fact that every bounded domain in $\mathbb{C}^{d}$ is Kobayashi hyperbolic. On the other hand, it is easy to check that $K_{\mathbb{C}^{d}} \equiv 0$ for all $d \geq 1$. We refer the interested reader to [14] (also see [12, Chapter 3]) for a comprehensive account on Kobayashi pseudo-distance. Now, we recall the following generalization of Liouville's theorem.

Result 2.1 Let $\mathscr{X}$ be a Kobayashi hyperbolic complex manifold and let $F: \mathbb{C}^{d} \longrightarrow$ $\mathscr{X}$ be a holomorphic map. Then $F$ is a constant function.

We are now in a befitting position to present

The proof of Lemma 1.1 Fix $\Omega \subset \mathbb{C}$ and $n \geq 2$. Now consider a point $W \in S_{n}(\Omega)$. Let $D$ be a diagonal matrix such that $\mathbf{c}(D)=\mathbf{c}(W)$. We know that there exists $C \in M_{n}(\mathbb{C})$ and a strictly upper triangular matrix $U$ such that

$$
W=\exp (-C)(D+U) \exp (C) .
$$

Now consider the map $f: \mathbb{C} \longrightarrow M_{n}(\mathbb{C})$ defined by

$$
f(\zeta):=\exp (-C \zeta)(D+\zeta U) \exp (C \zeta) \quad \forall \zeta \in \mathbb{C}
$$

Note that $\mathbf{c}(f(\zeta))=\mathbf{c}(D+\zeta U)=\mathbf{c}(D)$, hence $f(\mathbb{C}) \subset S_{n}(\Omega)$. Since $f$ is a nonconstant holomorphic map into $S_{n}(\Omega)$, by Result 2.1, $S_{n}(\Omega)$ cannot be Kobayashi hyperbolic. 
Recall, a complex manifold $\mathscr{X}$ is called taut if every sequence in $\mathcal{O}(\mathbb{D}, \mathscr{X}$ ) either has a convergent subsequence or a compactly divergent subsequence. It is a fact that every Kobayashi complete complex manifold is taut and every taut complex manifold is Kobayashi hyperbolic; the converse of both these facts do not hold. We now state the relevant results from the iteration theory of holomorphic self-maps on taut complex manifolds that we need later. Most of the material presented here is taken from Chapter 2.1 and Chapter 2.4 in Abate [2] (also, see Kobayashi [14]). We begin with stating a result that is due to $\mathrm{Wu}[21$, Theorem $\mathrm{C}]$.

Result 2.2 Let $\mathscr{X}$ be a taut complex manifold and let $f \in \mathcal{O}(\mathscr{X}, \mathscr{X})$ be such that $f\left(z_{0}\right)=z_{0}$ for some $z_{0} \in \mathscr{X}$. Then:

(a) the spectrum of the derivative of $f$ at $z_{0}, f^{\prime}\left(z_{0}\right)$, is contained in $\overline{\mathbb{D}}$.

(b) $f^{\prime}\left(z_{0}\right)=\mathbb{I}$ if and only if $f$ is the identity function.

(c) The tangent space $T_{z_{0}} \mathscr{X}$ admits a $f^{\prime}\left(z_{0}\right)$-invariant splitting $T_{z_{0}} \mathscr{X}=L_{N} \oplus L_{U}$ such that the spectrum of $\left.f^{\prime}\left(z_{0}\right)\right|_{L_{N}}$ is contained in $\mathbb{D}$, the spectrum of $\left.f^{\prime}\left(z_{0}\right)\right|_{L_{U}}$ is contained in $\partial \mathbb{D}$ and $\left.f^{\prime}\left(z_{0}\right)\right|_{L_{U}}$ is diagonalizable.

The subspace $L_{U}$ of $T_{z_{0}} \mathscr{X}$ is called the unitary space of $f$ at the fixed point $z_{0}$ and the subspace $L_{N}$ is called the nilpotent space of $f$ at $z_{0}$.

Before we state the next result, we need a definition. Let $\mathscr{X}$ be a complex manifold. A holomorphic retraction of $\mathscr{X}$ is a holomorphic map $\rho: \mathscr{X} \longrightarrow \mathscr{X}$ such that $\rho^{2}=$ $\rho$. A holomorphic retract of $\mathscr{X}$ is the image of $\mathscr{X}$ under a holomorphic retraction. It is known that any holomorphic retract of $\mathscr{X}$ is a closed complex submanifold of $\mathscr{X}$. We now state

Result 2.3 Let $\mathscr{X}$ be a taut complex manifold and $f \in \mathcal{O}(\mathscr{X}, \mathscr{X})$. Assume that the sequence $\left\{f^{k}\right\}$ of iterates of $f$ is not compactly divergent. Then there exist a complex submanifold $\mathscr{M}$ of $\mathscr{X}$ and a holomorphic retraction $\rho: \mathscr{X} \longrightarrow \mathscr{M}$ such that every limit point $h \in \mathcal{O}(\mathscr{X}, \mathscr{X})$ of $\left\{f^{k}\right\}$ is of the form

$$
h=\gamma \circ \rho,
$$

where $\gamma$ is an automorphism of $\mathscr{M}$. Moreover, even $\rho$ is a limit point of the sequence $\left\{f^{k}\right\}$.

The above result is due to Abate [1]. The manifold $\mathscr{M}$ above is called the limit manifold of $f$ and its dimension is called the limit multiplicity of $f$. If $f \in \mathcal{O}(\mathscr{X}, \mathscr{X})$ be such that $f\left(z_{0}\right)=z_{0}$ for some $z_{0} \in \mathscr{X}, \mathscr{X}$ being taut, then following is an easy consequence of the above results:

Corollary 2.4 Given $f \in \mathcal{O}(\mathscr{X}, \mathscr{X})$ with $f\left(z_{0}\right)=z_{0}$ for some $z_{0} \in \mathscr{X}$ and $\mathscr{X}$ being a taut complex manifold, the unitary space of $f$ at $z_{0}$ is the tangent space at $z_{0}$ of the limit manifold of $f$. In particular, the limit multiplicity of $f$ is the number of eigenvalues of $f^{\prime}\left(z_{0}\right)$ that belong to $\partial \mathbb{D}$ counted with multiplicity.

We also need a result due to Abate [1] that gives a characterization for the sequence $\left\{f^{k}\right\} \in \mathcal{O}(\mathscr{X}, \mathscr{X})$ to be convergent. 
Result 2.5 Let $\mathscr{X}$ be a taut complex manifold and let $f \in \mathcal{O}(\mathscr{X}, \mathscr{X})$. Then the sequence of iterates $\left\{f^{k}\right\}$ converges in $\mathcal{O}(\mathscr{X}, \mathscr{X})$ if and only if $f$ has a fixed point $z_{0} \in \mathscr{X}$ such that the spectrum of $f^{\prime}\left(z_{0}\right)$ is contained in $\mathbb{D} \cup\{1\}$.

We end this section with a result due to Vigué about the fixed-point set of a holomorphic self-map. Given $f \in \mathcal{O}(\mathscr{X}, \mathscr{X})$, we shall denote by $\operatorname{Fix}(f)$ the set of fixed points of $f$.

Result 2.6 (Vigué, [20]) Let $\mathscr{X}$ be a taut complex manifold, $f \in \mathcal{O}(\mathscr{X}, \mathscr{X})$. Then $\operatorname{Fix}(f)$ is a closed complex submanifold of $\mathscr{X}$. Moreover, for $x \in \operatorname{Fix}(f)$, we have

$$
T_{x}(\operatorname{Fix}(f))=\left\{\xi \in T_{x} \mathscr{X}: f^{\prime}(x) \xi=\xi\right\} .
$$

Also, see [14, Theorem 5.5.8] for details.

\section{Two Preliminary Lemmas}

In this section, we state two closely related lemmas. Lemma 3.3 is one of the key tools in the proof of the two main results of this paper. Both lemmas are simple once we appeal to a result by Zwonek. We begin by stating this result.

Result 3.1 (Zwonek, [23]) Let $\Omega \subset \mathbb{C}$ be a domain and let $n \in \mathbb{N}, n \geq 2$, be fixed. If $\#(\mathbb{C} \backslash \Omega) \geq 2 n$ then $\Sigma^{n}(\Omega)$ is Kobayashi complete. If $\#(\mathbb{C} \backslash \Omega)<2 n$ then $\Sigma^{n}(\Omega)$ contains a non-constant holomorphic image of $\mathbb{C}$ and thus $\Sigma^{n}(\Omega)$ is not Kobayashi hyperbolic.

Lemma 3.2 Consider a domain $\Omega \subset \mathbb{C}$ and $n \in \mathbb{N}, n \geq 2$, such that $\#(\mathbb{C} \backslash \Omega) \geq 2 n$. Let $\Psi$ be a holomorphic self-map of $S_{n}(\Omega)$. Then for every $W_{1}, W_{2} \in S_{n}(\Omega)$ such that $\mathbf{c}\left(W_{1}\right)=\mathbf{c}\left(W_{2}\right)$, we have $\mathbf{c}\left(\Psi\left(W_{1}\right)\right)=\mathbf{c}\left(\Psi\left(W_{2}\right)\right)$, where $\mathbf{c}: S_{n}(\Omega) \longrightarrow \Sigma^{n}(\Omega)$ is as defined in Sect. 1.

Proof Fix $W_{1}, W_{2} \in S_{n}(\Omega)$ such that $\mathbf{c}\left(W_{1}\right)=\mathbf{c}\left(W_{2}\right)$. We know that there exists $C \in M_{n}(\mathbb{C})$ and a strictly upper triangular matrix $U$ such that $W_{1}=\exp (-C)(D+$ $U) \exp (C)$, where $D$ is a diagonal matrix such that $\mathbf{c}(D)=\mathbf{c}\left(W_{1}\right)$. Now consider the map $f: \mathbb{C} \longrightarrow M_{n}(\mathbb{C})$ defined by

$$
f(\zeta):=\exp (-C \zeta)(D+\zeta U) \exp (C \zeta) \text { for all } \zeta \in \mathbb{C}
$$

Note that $\mathbf{c}(f(\zeta))=\mathbf{c}(D+\zeta U)=\mathbf{c}(D)$, hence $f(\mathbb{C}) \subset S_{n}(\Omega)$. This allows us to define the map $g(\zeta):=\mathbf{c} \circ \Psi \circ f(\zeta)$ for all $\zeta \in \mathbb{C}$. Note that $g$ is a holomorphic map from $\mathbb{C}$ to $\Sigma^{n}(\Omega)$. Since $\#(\mathbb{C} \backslash \Omega) \geq 2 n$, by Result 3.1, it follows that $\Sigma^{n}(\Omega)$ is Kobayashi hyperbolic. Then using Result 2.1, we get that $g$ is a constant function. Hence

$$
\mathbf{c}(\Psi(D))=g(0)=g(1)=\mathbf{c}\left(\Psi\left(W_{1}\right)\right)
$$


Proceeding similarly we get $\mathbf{c}(\Psi(D))=\mathbf{c}\left(\Psi\left(W_{2}\right)\right)$ whence $\mathbf{c}\left(\Psi\left(W_{1}\right)\right)=\mathbf{c}\left(\Psi\left(W_{2}\right)\right)$. Since the choice of $W_{1}, W_{2} \in S_{n}(\Omega)$ (satisfying $\mathbf{c}\left(W_{1}\right)=\mathbf{c}\left(W_{2}\right)$ ) was arbitrary, the lemma follows.

The above lemma is motivated from that of [17, Theorem 1] by Ransford-White. It also appeared in [9] but we present the proof here for completeness. Further, with the help of this lemma, we prove the following result:

Lemma 3.3 Consider a domain $\Omega \subset \mathbb{C}$ and $n \in \mathbb{N}, n \geq 2$, such that $\#(\mathbb{C} \backslash \Omega) \geq 2 n$. Let $\Psi$ be a holomorphic self-map of $S_{n}(\Omega)$. Then there exists a unique holomorphic self-map $G_{\Psi}$ of $\Sigma^{n}(\Omega)$ such that $G_{\Psi} \circ \mathbf{c}=\mathbf{c} \circ \Psi$ (also see Fig. 1).

Proof Consider a relation $G_{\Psi}$ from $\Sigma^{n}(\Omega)$ into $\Sigma^{n}(\Omega)$ defined by

$$
G_{\Psi}(z):=\mathbf{c} \circ \Psi \circ \mathbf{c}^{-1}(z) \quad \forall z \in \Sigma^{n}(\Omega) .
$$

From Lemma 3.2, it follows that for each $z \in \Sigma^{n}(\Omega), G_{\Psi}(z)$ is a singleton. Hence $G_{\Psi}: \Sigma^{n}(\Omega) \longrightarrow \Sigma^{n}(\Omega)$ is a well-defined map that satisfies the relation $G_{\Psi} \circ \mathbf{c}=$ c $\circ \Psi$. The lemma now follows once we prove the following claim:

Claim $G_{\Psi}$ is holomorphic.

To see this, fix $z \in \Sigma^{n}(\Omega)$. Now consider the polynomial $P_{z}(t):=t^{n}+$ $\sum_{j=1}^{n}(-1)^{j} z_{j} t^{n-j}$ and define the map $\kappa: \Sigma^{n}(\Omega) \longrightarrow M_{n}(\mathbb{C})$ by setting

$$
\kappa(z):=\mathrm{C}\left(P_{z}\right)
$$

where $\mathrm{C}\left(P_{z}\right)$ denotes the companion matrix of the polynomial $P_{z}$. Recall, given a monic polynomial of degree $k$ of the form $p(t)=t^{k}+\sum_{j=1}^{k} a_{j} t^{k-j}$, where $a_{j} \in \mathbb{C}$, the companion matrix of $p$ is the matrix $C(p) \in M_{k}(\mathbb{C})$ given by

$$
C(p):=\left[\begin{array}{cccc}
0 & & & -a_{k} \\
1 & 0 & & -a_{k-1} \\
& \ddots & \ddots & \vdots \\
0 & & 1 & -a_{1}
\end{array}\right]_{k \times k} .
$$

It is a fact that $\mathbf{c}(\mathrm{C}(p))=\left(a_{1}, \ldots, a_{k}\right)$. From this, it follows that $\kappa$ is holomorphic and $\mathbf{c} \circ \kappa=\mathbb{I}$ on $\Sigma^{n}(\Omega)$. This, in particular, implies that $\kappa(z) \in \mathbf{c}^{-1}(z)$. Applying Lemma 3.2 again, we see that $\mathbf{c} \circ \Psi \circ \mathbf{c}^{-1}(z)=\mathbf{c} \circ \Psi \circ \kappa(z)$, i.e. $G_{\Psi}(z)=\mathbf{c} \circ \Psi \circ \kappa(z)$. Since each of the maps $\mathbf{c}, \Psi, \kappa$ are holomorphic, the claim follows.

\section{Preparations for the Proof of Theorem 1.2}

In this section, we devise certain ingredients that play a crucial role in the proof of Theorem 1.2. We first show that given a point $A \in M_{n}(\mathbb{C})\left(\equiv \mathbb{C}^{n^{2}}\right)$ there is a polydisc centred at $A$ on which the map c could be decomposed. We also describe the utility of this decomposition to our proof of Theorem 1.2. In what follows, given integers $j<k,[j, \ldots, k]$ will denote the set of integers $\{j, j+1, \ldots, k\}$. 


\subsection{Local Decomposition of c}

Recall that given $x \in \mathbb{C}^{n}, P_{x}(t)$ is the polynomial $t^{n}+\sum_{j=1}^{n}(-1)^{j} x_{j} t^{n-j}$. Given $n \geq 2$, suppose there exist positive integers $n_{i}, i \in[1, \ldots, m]$ such that $\sum_{i=1}^{m} n_{i}=n$. Consider the map $\tau: \prod_{i=1}^{m} \Sigma^{n_{i}}(\Omega) \longrightarrow \Sigma^{n}(\Omega)$ defined by

$$
\tau\left(x_{1}, \ldots, x_{m}\right)=y \text {, where } y \text { satisfies } P_{y}(t)=\prod_{i=1}^{m} P_{x_{i}}(t) \text {. }
$$

Note that $\tau$ is a holomorphic surjective map. We now state the result regarding the local decomposition of $\mathbf{c}$.

Lemma 4.1 Let $A \in S_{n}(\Omega), n \geq 2$, and write $\sigma(A):=\left\{\lambda_{1}, \ldots, \lambda_{m}\right\}$ such that for each $i \in[1, \ldots, m], n_{i}$ is the algebraic multiplicity of $\lambda_{i}$. Then there exists $a$ $\delta>0$ such that on the polydisc $P(A ; \delta)$, the map $\mathbf{c}$ decomposes as $\mathbf{c}=\tau \circ \theta$, where $\theta: P(A ; \delta) \longrightarrow \prod_{i=1}^{m} \Sigma^{n_{i}}(\Omega)$ is a holomorphic open map and $\tau: \prod_{i=1}^{m} \Sigma^{n_{i}}(\Omega) \longrightarrow$ $\Sigma^{n}(\Omega)$, as defined above, is a biholomorphism from $\theta(P(A ; \delta))$ onto $\mathbf{c}(P(A ; \delta))$.

Proof Choose an $r>0$ such that $r<\min \left\{\left|\lambda_{i}-\lambda_{j}\right| / 2: i, j \in[1, \ldots, m], i \neq\right.$ $j\}$ and the discs $\mathbb{D}\left(\lambda_{i} ; r\right):=\left\{\zeta \in \mathbb{C}:\left|\zeta-\lambda_{i}\right|<r\right\}$ are contained in $\Omega$. Now using the continuity of the map $\mathbf{c}$ and the fact that the roots of a polynomial - as a function of its coefficients - vary continuously, we can find a $\delta>0$ such that for any $W \in P(A ; \delta) \subset \mathbb{C}^{n^{2}}$ the number of eigenvalues of $W$ in $\mathbb{D}\left(\lambda_{i} ; r\right)$, counted with multiplicity, is $n_{i}$ for all $i \in[1, \ldots, m]$. Given $W \in P(A ; \delta)$, denote by $\sigma_{i}(W)^{\bullet}$ a list of eigenvalues of $\sigma(W)$ that lie in the disc $\mathbb{D}\left(\lambda_{i} ; r\right)$ and are repeated with their multiplicity. Note that the number of elements in $\sigma_{i}(W)^{\bullet}$ is $n_{i}$ for each $i$. Now, we define the map $\theta: P(A ; \delta) \longrightarrow \prod_{i=1}^{m} \Sigma^{n_{i}}(\Omega)$ by

$$
\begin{aligned}
& \theta(W)=\left(\theta_{1}(W), \ldots, \theta_{m}(W)\right), \text { where each } \theta_{i}(W) \text { satisfy } \\
& P_{\theta_{i}(W)}(t)=\prod_{\mu \in \sigma_{i}(W)}(t-\mu)
\end{aligned}
$$

It is not difficult to see that $\mathbf{c}$ and $\theta$ are open maps-see Sect. 7 for details. It follows from (4.1) and (4.2) that

$$
\tau\left(\theta_{1}(W), \ldots, \theta_{m}(W)\right)=\mathbf{c}(W) \text { for all } W \in P(A ; \delta)
$$

We now show that $\tau$ is a biholomorphism from the open set $\theta(P(A ; \delta))$ onto the open set $\mathbf{c}(P(A ; \delta))$. Notice we only need to show that $\tau$ is injective on $\theta(P(A ; \delta))$. Suppose

$$
\tau\left(x_{1}, \ldots, x_{m}\right)=\tau\left(y_{1}, \ldots, y_{m}\right),
$$


where $\left(x_{1}, \ldots, x_{m}\right)=\theta\left(W_{1}\right)$ and $\left(y_{1}, \ldots, y_{m}\right)=\theta\left(W_{2}\right)$ for some $W_{1}, W_{2} \in$ $P(A ; \delta)$. It follows from the definition of $\tau$ and (4.4) that

$$
\prod_{j=1}^{m} P_{x_{j}}(t)=\prod_{j=1}^{m} P_{y_{j}}(t) .
$$

Fix a $j \in[1, \ldots, m]$. Since $x_{j}=\theta_{j}\left(W_{1}\right)$ and $W_{1} \in P(A ; \delta)$, the zeros of $P_{x_{j}}(t)$ lie in the disc $\mathbb{D}\left(\lambda_{j} ; r\right)$. Similarly for any $k \neq j$-since $y_{k}=\theta_{k}\left(W_{2}\right)$ and $W_{2} \in P(A ; \delta)$ the zeros of the polynomial $P_{y_{k}}(t)$ lie in the $\operatorname{disc} \mathbb{D}\left(\lambda_{k} ; r\right)$. As $\mathbb{D}\left(\lambda_{j} ; r\right) \cap \mathbb{D}\left(\lambda_{k} ; r\right)=\varnothing$ for $k \neq j$ whence the zeros of the polynomial $P_{x_{j}}(t)$ are also the zeros of $P_{y_{j}}(t)$. Reversing this argument we see that the zeros of $P_{x_{j}}(t)$ and $P_{y_{j}}(t)$ coincide. Hence $x_{j}=y_{j}$ for each $j \in[1, \ldots, m]$ showing the injectivity of $\tau$ on $\theta(P(A ; \delta))$.

The holomorphicity of the map $\theta$ on $P(A ; \delta)$ now follows from (4.3) together with the fact that $\tau$ is a biholomorphism from $\theta(P(A ; \delta))$ onto $\mathbf{c}(P(A ; \delta))$.

We now present a lemma that paves the way towards the proof of Theorem 1.2.

Lemma 4.2 Given $n \in \mathbb{N}, n \geq 2$, and a domain $\Omega$ in $\mathbb{C}$ satisfying $\#(\mathbb{C} \backslash \Omega) \geq 2 n$. Let $\Psi \in \mathcal{O}\left(S_{n}(\Omega), S_{n}(\Omega)\right)$ such that $\Psi(A)=A, \Psi^{\prime}(A)=\mathbb{I}$ for some $A \in S_{n}(\Omega)$. Then there exist neighbourhoods $\mathcal{V}_{j} \subset P(A ; \delta), j=1,2$, of $A$ satisfying $\mathcal{V}_{2}=\Psi\left(\mathcal{V}_{1}\right)$ such that if we define $F_{\Psi}: \theta\left(\mathcal{V}_{1}\right) \longrightarrow \theta\left(\mathcal{V}_{2}\right)$ by

$$
F_{\Psi} \equiv\left(\left.\tau\right|_{\theta\left(\mathcal{V}_{2}\right)}\right)^{-1} \circ G_{\Psi} \circ \tau
$$

where $\theta, \tau, P(A ; \delta)$ are as in Lemma 4.1 and $G_{\Psi}$ is as in Lemma 3.3 then the following diagram is commutative:

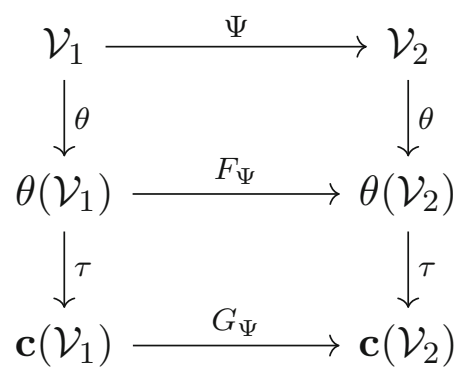

Fig. $2 G_{\Psi}$ and $F_{\Psi}$ are conjugates.

Proof Since $\Psi(A)=A$ and $\Psi^{\prime}(A)=\mathbb{I}$, the inverse function theorem implies that there are neighbourhoods $\mathcal{V}_{1}, \mathcal{V}_{2}$ of $A$ that are contained in the polydisc $P(A ; \delta)$ such that $\Psi\left(\mathcal{V}_{1}\right)=\mathcal{V}_{2}$. Observe that we only need to show that on $\mathcal{V}_{1}$,

$$
F_{\Psi} \circ \theta=\theta \circ \Psi \text {. }
$$


Notice, by the definition of $F_{\Psi}$, we have

$$
F_{\Psi} \circ \theta=\left[\left(\left.\tau\right|_{\theta\left(\mathcal{V}_{2}\right)}\right)^{-1} \circ G_{\Psi} \circ \tau\right] \circ \theta=\left(\left.\tau\right|_{\theta\left(\mathcal{V}_{2}\right)}\right)^{-1} \circ G_{\Psi} \circ \mathbf{c} .
$$

In the above we have used the identity $\tau \circ \theta=\mathbf{c}$ on $P(A ; \delta)$. Now, since $G_{\Psi} \circ \mathbf{c}=\mathbf{c} \circ \Psi$, the above equation becomes

$$
F_{\Psi} \circ \theta=\left(\left.\tau\right|_{\theta\left(\mathcal{V}_{2}\right)}\right)^{-1} \circ \mathbf{c} \circ \Psi
$$

Now, on $\mathcal{V}_{2}$, we have $\theta=\left(\left.\tau\right|_{\theta\left(\mathcal{V}_{2}\right)}\right)^{-1} \circ$ c. Putting this into the above equation gives us the desired equality.

The following two points encapsulates the importance of Lemma 4.2 and the commutative diagram therein in our proof of Theorem 1.2:

- The main goal in our proof of Theorem 1.2-when $A$ is diagonalizable-will be to prove that the trace of $G_{\Psi}^{\prime}$ at $a=\mathbf{c}(A)$ is $n$. Since the map $G_{\Psi}$ and $F_{\Psi}$ are locally biholomorphic conjugates of each other, it is sufficient to show that there is a basis $\mathfrak{B}$ of $\mathbb{C}^{n}$ such that the trace of $F_{\Psi}^{\prime}$ at $a^{*}=\theta(A)$ with respect to $\mathfrak{B}$ is $n$.

- The commutativity of the upper-half part of the above diagram enables us in computing the trace of $F_{\Psi}^{\prime}$ at $a^{*}$ with respect to an appropriately chosen basis $\mathfrak{B}$ as mentioned above. In fact, in the next subsection, we shall construct a basis $\mathfrak{B}$ and derive a very important information regarding the trace of certain diagonal blocks of $\left[F_{\Psi}^{\prime}\left(a^{*}\right)\right]_{\mathfrak{B}}:=$ the derivative matrix of $F_{\Psi}$ at $a^{*}$ with respect to $\mathfrak{B}$.

\subsection{An Important Proposition}

We continue with the set-up as in Lemma 4.2. Assuming that the matrix $A$ in the aforementioned lemma is a diagonal matrix, we derive an important information regarding the trace of $\left[F_{\Psi}^{\prime}\left(a^{*}\right)\right]_{\mathfrak{B}}$ with respect to an appropriately chosen basis $\mathfrak{B}$. For simplicity, we shall write the maps $F_{\Psi}, G_{\Psi}$ as $F, G$, respectively. Note the map $F: \theta\left(\mathcal{V}_{1}\right) \longrightarrow \theta\left(\mathcal{V}_{2}\right)$, where $\mathcal{V}_{1}$ and $\mathcal{V}_{2}$ are as in Lemma 4.2, can be written as $F=\left(F_{1}, \ldots, F_{m}\right)$ such that $F_{i}\left(\theta\left(\mathcal{V}_{1}\right)\right) \subset \Sigma^{i}(\Omega)$ for all $i \in[1, \ldots, m]$. Also, if we let $\mathfrak{A}_{i}:=\left\{\mathbf{e}_{1}^{i}, \ldots, \mathbf{e}_{n_{i}}^{i}\right\}$ denote the standard basis of $\mathbb{C}^{n_{i}}$ then write $F_{i}:=\sum_{j=1}^{n_{i}} F_{i, j} \mathbf{e}_{j}^{i}$ on $\theta\left(\mathcal{V}_{1}\right)$. The following result by Sun is at the heart of the proof of the main result of this subsection:

Result 4.3 (Paraphrasing of Corollary 1.2 in [19]) Let $X \in M_{n}(\mathbb{C})$ be a normal matrix with $\sigma(X)^{\bullet}=\left\{\zeta_{1}, \ldots, \zeta_{n}\right\}$. Here $\sigma(X)^{\bullet}$ denotes a list of eigenvalues of $X$ repeated according to their multiplicity. Let $Y$ be any other matrix with $\sigma(Y)^{\bullet}=\left\{\xi_{1}, \ldots, \xi_{n}\right\}$. Then there exists a permutation $\pi$ of $[1, \ldots, n]$ such that

$$
\max \left\{\left|\xi_{\pi(j)}-\zeta_{j}\right|: j \in[1, \ldots, n]\right\} \leq n\|X-Y\|_{\mathrm{op}}
$$

where $\|\cdot\|_{\mathrm{op}}$ denotes the operator norm of a matrix considered as a bounded linear operator on the Hilbert space $\left(\mathbb{C}^{n},\|\cdot\|_{2}\right)$. 
We are now in a position to state our main result of this subsection. (In what follows,

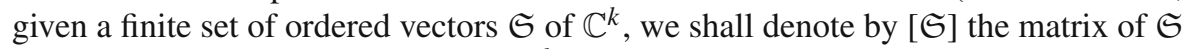
with respect to the standard basis of $\mathbb{C}^{k}$ ).

Proposition 4.4 Suppose the matrix A in Lemma 4.2 is a diagonal matrix with the $i_{0}$ th eigenvalue $\lambda_{i_{0}}=0$. Then there is a basis $\mathfrak{B}_{i}, i \in[1, \ldots, m]$ of $\mathbb{C}^{n_{i}}$ such that the basis $\mathfrak{B}$ of $\mathbb{C}^{n}$ defined by

$$
[\mathfrak{B}]=\left[\mathfrak{B}_{1}\right] \oplus \cdots \oplus\left[\mathfrak{B}_{m}\right]
$$

has the property that the $i_{0}$ th diagonal block of size $n_{i_{0}} \times n_{i_{0}}$ of the matrix $\left[F^{\prime}\left(a^{*}\right)\right]_{\mathfrak{B}}$ has trace $n_{i_{0}}$.

Proof We first construct the basis $\mathfrak{B}_{i}:=\left\{\mathbf{v}_{j}^{i} \in \mathbb{C}^{n_{i}}: j \in\left[1, \ldots, n_{i}\right]\right\}$ for each $i \in[1, \ldots, m]$. These are defined by the equation

$$
P_{\theta_{i}(A)+\eta \mathbf{v}_{j}^{i}}(t)=\left(t-\lambda_{i}\right)^{n_{i}}+\eta\left(t-\lambda_{i}\right)^{n_{i}-j} \quad \forall j \in\left[1, \ldots, n_{i}\right],
$$

where $P$ and $\theta_{i}$ 's are as in Sect. 4.1. It is easy to see that

$$
\mathbf{v}_{j}^{i}:= \begin{cases}\left(0, \ldots, 0,1, \pi_{n_{i}-j}\left(\lambda_{i}, \ldots, \lambda_{i}\right)\right), & \text { if } j \in\left[1, \ldots, n_{i}-1\right] \\ (0, \ldots, 0,1), & \text { otherwise }\end{cases}
$$

where $\pi_{n_{i}-j}: \mathbb{C}^{n_{i}-j} \longrightarrow \mathbb{C}^{n_{i}-j}$ is the symmetrization map. Notice $\mathfrak{B}_{i}$ is a set of $n_{i}$ linearly independent vectors and hence $\mathfrak{B}_{i}$ forms a basis of $\mathbb{C}^{n_{i}}$. Since $\lambda_{i_{0}}=0$, we also see that $\mathfrak{B}_{i_{0}}=\mathfrak{A}_{i_{0}}$. For each $i \in[1, \ldots, m]$, we write

$$
\theta_{i}=\sum_{j=1}^{n_{i}} \widehat{\theta}_{i, j} \mathbf{v}_{j}^{i} \text { and } F_{i}=\sum_{j=1}^{n_{i}} \widehat{F}_{i, j} \mathbf{v}_{j}^{i} .
$$

Since $\mathfrak{B}_{i_{0}}=\mathfrak{A}_{i_{0}}$, we have $\widehat{\theta}_{i_{0}, j}=\theta_{i_{0}, j}$ and $\widehat{F}_{i_{0}, j}=F_{i_{0}, j}$ for all $j \in\left[1, \ldots, n_{i_{0}}\right]$. Denote by $\mathbf{V}_{j}^{i_{0}}:=\left(\mathbf{V}_{j, 1}^{i_{0}}, \ldots, \mathbf{V}_{j, i}^{i_{0}}, \ldots, \mathbf{V}_{j, m}^{i_{0}}\right) \in \prod_{i=1}^{m} \mathbb{C}^{n_{i}}$ such that $\mathbf{V}_{j, i}^{i_{0}}=\mathbf{0}$ when $i \neq i_{0}$ and $\mathbf{V}_{j, i_{0}}^{i_{0}}=\mathbf{v}_{j}^{i_{0}}$.

\section{Claim}

$$
\left[\frac{\partial \widehat{F}_{i_{0}, j}}{\partial \mathbf{V}_{k}^{i_{0}}}\left(a^{*}\right)\right]=\left[\frac{\partial F_{i_{0}, j}}{\partial \mathbf{E}_{k}^{i_{0}}}\left(a^{*}\right)\right]=\mathbb{I}_{n_{i_{0}}}+N
$$

where $N$ is an upper triangular nilpotent matrix and $\mathbb{I}_{n_{i_{0}}}$ is the identity matrix of order $n_{i_{0}}$. Also, $\mathbf{E}_{k}^{i_{0}} \in \prod_{i=1}^{m} \mathbb{C}^{n_{i}}$ is a vector whose $i_{0}$ th component is $\mathbf{e}_{k}^{i_{0}}$ and every other component is the zero vector.

To establish the claim, we begin with the observation

$$
\frac{\partial F_{i_{0}, j}}{\partial \mathbf{E}_{k}^{i_{0}}}\left(a^{*}\right)=\lim _{\epsilon \rightarrow 0} \frac{F_{i_{0}, j}\left(a^{*}+\epsilon \mathbf{E}_{k}^{i_{0}}\right)-F_{i_{0}, j}\left(a^{*}\right)}{\epsilon} .
$$


Now, by Lemma 4.2, $F_{i_{0}, j}=\theta_{i_{0}, j} \circ \Psi \circ \theta^{-1}$. Substituting this together with the observation that $\theta_{i_{0}, j}(A)=0$ gives us

$$
\frac{\partial F_{i_{0}, j}}{\partial \mathbf{E}_{k}^{i_{0}}}\left(a^{*}\right)=\lim _{\epsilon \rightarrow 0} \frac{\theta_{i_{0}, j} \circ \Psi \circ \theta^{-1}\left(a^{*}+\epsilon \mathbf{E}_{k}^{i_{0}}\right)}{\epsilon} .
$$

Let us now write $\mathbb{I}_{n}=\mathbb{I}_{n_{1}} \oplus \cdots \oplus \mathbb{I}_{n_{m}}$. Let $D_{k}^{i_{0}} \in M_{n_{i_{0}}}(\mathbb{C})$ be the diagonal matrix defined by

$$
D_{k}^{i_{0}}=\operatorname{diag}\left[\omega_{1}, \omega_{2}, \ldots, \omega_{k}, 0, \ldots, 0\right], \quad k \in\left[1, \ldots, n_{i_{0}}\right]
$$

where $\omega_{j}$ 's are the roots of the equation $x^{k}+1=0$. Consider the matrix $D_{k}=$ $\oplus_{i=1}^{m} W_{i}$, where $W_{i}=0 \in M_{n_{i}}(\mathbb{C})$, if $i \neq i_{0}$ and $W_{i_{0}}=D_{k}^{i_{0}}$. Observe that (when $\epsilon$ is sufficiently small)

$$
\theta_{i}\left(A+\epsilon^{1 / k} D_{k}\right):= \begin{cases}\theta_{i}(A), & \text { if } i \neq i_{0} \\ \epsilon \mathbf{e}_{k}^{i_{0}}, & \text { otherwise }\end{cases}
$$

Hence $\theta\left(A+\epsilon^{1 / k} D_{k}\right)=a^{*}+\epsilon \mathbf{E}_{k}^{i_{0}}$ for all $k \in\left[1, \ldots, n_{i_{0}}\right]$. Substituting this into (4.7) we get

$$
\frac{\partial F_{i_{0}, j}}{\partial \mathbf{E}_{k}^{i_{0}}}\left(a^{*}\right)=\lim _{\epsilon \rightarrow 0} \frac{\theta_{i_{0}, j} \circ \Psi\left(A+\epsilon^{1 / k} D_{k}\right)}{\epsilon}=\lim _{s \rightarrow 0} \frac{\theta_{i_{0}, j} \circ \Psi\left(A+s D_{k}\right)}{s^{k}} .
$$

Since $\Psi(A)=A$ and $\Psi^{\prime}(A)=\mathbb{I}$, for small enough $s$ we can write

$$
\Psi\left(A+s D_{k}\right)=A+s D_{k}+\sum_{j \geq 2} B_{j} s^{j},
$$

where $B_{j} \in M_{n}(\mathbb{C}), j \geq 2$. This, in particular, implies that

$$
\left\|\Psi\left(A+s D_{k}\right)-\left(A+s D_{k}\right)\right\|_{\mathrm{op}}=s^{2} M(s)
$$

where $M(s)$ is a continuous function in a neighbourhood of 0 . Now, when $s$ is sufficiently small both $\Psi\left(A+s D_{k}\right)$ and $\left(A+s D_{k}\right)$ lie in $\mathcal{V}_{2} \subset P(A ; \delta)$ and $\mathcal{V}_{1} \subset P(A ; \delta)$, respectively. Furthermore, the non-zero eigenvalues of $A+s D_{k}$ that lie in the disc $\mathbb{D}\left(\lambda_{i_{0}} ; r\right) \equiv \mathbb{D}(0 ; r)$ are $s \omega_{1}, \ldots, s \omega_{k}$. Notice that the matrices $A+s D_{k}$ are all diagonal matrices. So if we denote by $\mu_{j}^{i_{0}}(s), j \in\left[1, \ldots, n_{i_{0}}\right]$ the eigenvalues of $\Psi\left(A+s D_{k}\right)$ that lie in the $\operatorname{disc} \mathbb{D}(0 ; r)$ then by Result 4.3 , there exists $\zeta_{j}(s) \in \mathbb{D}$, $j \in\left[1, \ldots, n_{i_{0}}\right]$ such that

$$
\mu_{j}^{i_{0}}(s)= \begin{cases}s \omega_{j}+\zeta_{j}(s) n s^{2} M(s), & \text { if } j \in[1, \ldots, k] \\ \zeta_{j}(s) n s^{2} M(s), & j \in\left[k+1, \ldots, n_{i_{0}}\right]\end{cases}
$$


For a fixed $j \geq 1$, let $\mathscr{I}_{j}$ be the collection of all possible subsets of $\left\{1,2, \ldots, n_{i_{0}}\right\}$ of cardinality $j$ and let

$$
\mu_{I}(s)=\mu_{i_{1}}(s) \mu_{i_{2}}(s) \ldots \mu_{i_{j}}(s), \text { where } I=\left\{i_{1}, i_{2}, \ldots, i_{j}\right\} \in \mathscr{I}_{j} .
$$

Then by definition

$$
\theta_{i_{0}, j} \circ \Psi\left(A+s D_{k}\right)=\sum_{I \in \mathscr{I}_{j}} \mu_{I}(s)
$$

Now note that for $j>k, \mu_{I}(s)=s^{j+1} h_{I}(s)$, where $h_{I}(s)$ are continuous functions in $s$ for every $I \in \mathscr{I}_{j}$. However, for $j=k, \mu_{I}(s)=s^{j}+s^{j+1} h_{I}(s)$ only if $I=$ $\{1,2, \ldots, j\}$ and $\mu_{I}(s)=s^{j+1} h_{I}(s)$ otherwise. Thus we have

$$
\frac{\partial F_{i_{0}, j}}{\partial \mathbf{E}_{k}^{i_{0}}}\left(a^{*}\right)=\lim _{s \rightarrow 0} \frac{\theta_{i_{0}, j} \circ \Psi\left(A+s D_{k}\right)}{s^{k}}= \begin{cases}1 & j=k \\ 0 & j>k\end{cases}
$$

which establishes the claim and consecutively proves our proposition.

\subsection{Translation by a Scalar Matrix}

The purpose of this subsection is to devise a translation trick which is another main tool in computing the trace of the derivative of the map $F_{\Psi}$ as in Lemma 4.2. For this purpose, given $\lambda \in \mathbb{C}$, define the translation $L_{\lambda}: M_{n}(\mathbb{C}) \longrightarrow M_{n}(\mathbb{C})$ by $L_{\lambda}(W):=W-\lambda \mathbb{I}$. Notice $\left(L_{\lambda}\right)^{-1}=L_{-\lambda}$. Furthermore, if $\Omega \subseteq \mathbb{C}$ be any domain then $L_{\lambda}\left(S_{n}(\Omega)\right)=S_{n}\left(\Omega_{\lambda}\right)$, where $\Omega_{\lambda}=\{z-\lambda: z \in \Omega\}$. Note that if $\Omega$ satisfies the cardinality condition $\#(\mathbb{C} \backslash \Omega) \geq 2 n$ then so does $\Omega_{\lambda}$. Observe the map $L_{-\lambda}$ introduces a map $G_{-\lambda}: \Sigma^{n}\left(\Omega_{\lambda}\right) \longrightarrow \Sigma^{n}(\Omega)$ such that $G_{-\lambda} \circ \mathbf{c}=\mathbf{c} \circ L_{-\lambda}$, i.e. the following diagram commutes (Fig. 3):

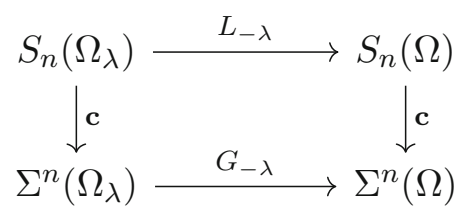

Fig. $3 L_{-\lambda}$ induces $G_{-\lambda}$

In fact, $G_{-\lambda}\left(\pi_{n}\left(z_{1}, \ldots, z_{n}\right)\right)=\pi_{n}\left(z_{1}+\lambda, \ldots, z_{n}+\lambda\right)$, where $\pi_{n}: \mathbb{C}^{n} \longrightarrow \mathbb{C}^{n}$ is the symmetrization map. Notice also that $G_{-\lambda}$ is a biholomorphism, its inverse $G_{\lambda}$ is defined by $G_{\lambda}\left(\pi_{n}\left(z_{1}, \ldots, z_{n}\right)\right)=\pi_{n}\left(z_{1}-\lambda, \ldots, z_{n}-\lambda\right)$.

We now define $\mathcal{V}_{j, \lambda}=L_{\lambda}\left(\mathcal{V}_{j}\right), j=1,2$, where $\mathcal{V}_{1}, \mathcal{V}_{2}$ are as in Lemma 4.2. Then we have the following commutative diagram (Fig. 4): 


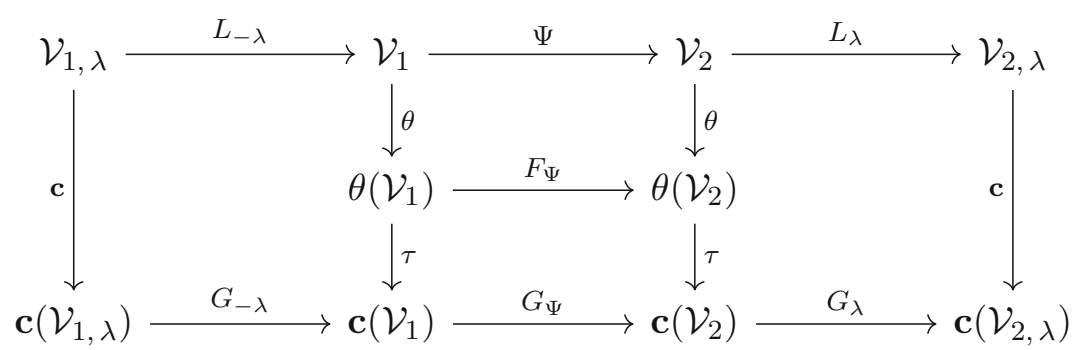

Fig. 4 Translation diagram 1.

Observe that $\mathcal{V}_{j, \lambda}$ are neighbourhoods of $A_{\lambda}:=A-\lambda \mathbb{I}$ of the same type as $\mathcal{V}_{j}$ 's are that of $A$. In other words, if we take $B \in \mathcal{V}_{j, \lambda}$ then the spectrum of $B$ is contained in the union of disjoint discs $\mathbb{D}\left(\lambda_{i}-\lambda, r\right)$ that are centred at $\lambda_{i}-\lambda$ with radius $r$, $i \in[1, \ldots, m]$, where $r>0$ is chosen as in the proof of Lemma 4.1. Moreover, the number of eigenvalues of $B$, counted with multiplicity, that lie in the disc $\mathbb{D}\left(\lambda_{i}-\lambda, r\right)$ is $n_{i}, i \in[1, \ldots, m]$. Proceeding exactly as in the proof of Lemmas 4.1 and 4.2, we see that there are maps $\theta_{\lambda}, \tau_{\lambda}$ and $F_{-\lambda}, F_{\lambda}$ such that the diagram in Fig. 5 is commutative.

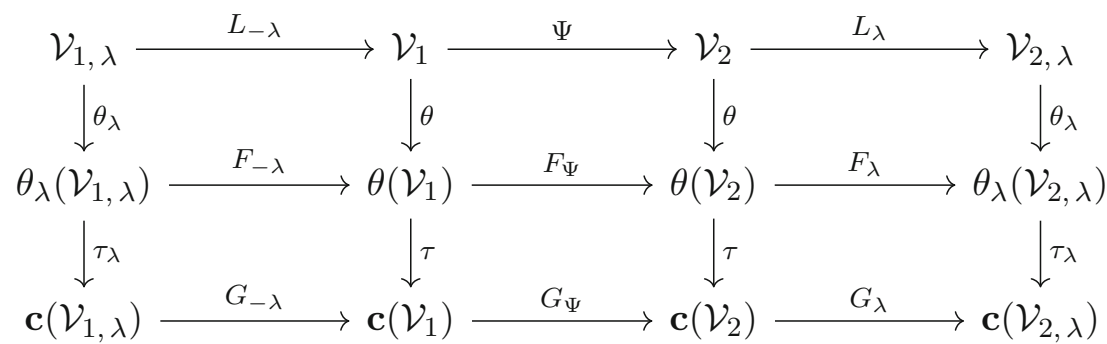

Fig. 5 Translation diagram 2.

Define the maps $\Psi_{\lambda}:=L_{\lambda} \circ \Psi \circ L_{-\lambda}, F_{\Psi_{\lambda}}:=F_{\lambda} \circ F_{\Psi} \circ F_{-\lambda}$ and $G_{\Psi_{\lambda}}:=$ $G_{\lambda} \circ G_{\Psi} \circ G_{-\lambda}$ and set $a_{\lambda}^{*}:=\theta_{\lambda}\left(A_{\lambda}\right)$. The following lemma is the translation trick that we alluded to in the start of this subsection:

Lemma 4.5 Let $\mathfrak{B}_{\lambda, i}, \mathfrak{B}_{0, i}$ be two bases of $\mathbb{C}^{n_{i}}, i \in[1, \ldots, m]$ and consider the bases $\mathfrak{B}_{\lambda}, \mathfrak{B}_{0}$ of $\mathbb{C}^{n}$ that are defined by

$$
\left[\mathfrak{B}_{\lambda}\right]=\left[\mathfrak{B}_{\lambda, 1}\right] \oplus \cdots \oplus\left[\mathfrak{B}_{\lambda, m}\right] \text { and }\left[\mathfrak{B}_{0}\right]=\left[\mathfrak{B}_{0,1}\right] \oplus \cdots \oplus\left[\mathfrak{B}_{0, m}\right] \text {. }
$$

Then the ith diagonal block of size $n_{i} \times n_{i}$ of the matrices $\left[F_{\Psi_{\lambda}}^{\prime}\left(a_{\lambda}^{*}\right)\right]_{\mathfrak{B}_{\lambda}}$ and $\left[F_{\Psi}^{\prime}\left(a^{*}\right)\right]_{\mathfrak{B}_{0}}$ are similar for every $i \in[1, \ldots, m]$.

Proof Since $F_{\Psi_{\lambda}}=F_{\lambda} \circ F_{\Psi} \circ F_{-\lambda}$ on $\theta_{\lambda}\left(\mathcal{V}_{1, \lambda}\right)$, by chain rule, we get

$$
\left[F_{\Psi_{\lambda}}^{\prime}\left(a_{\lambda}^{*}\right)\right]_{\mathfrak{B}_{\lambda}}=\left[F_{\lambda}^{\prime}\left(a_{\lambda}^{*}\right)\right]_{\mathfrak{B}_{0}}^{\mathfrak{B}_{\lambda}} \cdot\left[F_{\Psi}^{\prime}\left(a^{*}\right)\right]_{\mathfrak{B}_{0}} \cdot\left[F_{-\lambda}^{\prime}\left(a_{\lambda}^{*}\right)\right]_{\mathfrak{B}_{\lambda}}^{\mathfrak{B}_{0}} .
$$


As noted in the beginning of this section $G_{\lambda}, G_{-\lambda}$ are biholomorphisms and consequently, so are $F_{\lambda}, F_{-\lambda}$. Also, observe if we write $F_{\lambda}=\left(F_{\lambda, 1}, \ldots, F_{\lambda, m}\right)$ and $F_{-\lambda}=\left(F_{-\lambda, 1}, \ldots, F_{-\lambda, m}\right)$ then for every $x=\left(x_{1}, \ldots, x_{m}\right)$ and $i \in[1, \ldots, m]$, the values $F_{\lambda, i}(x), F_{-\lambda, i}(x)$ are independent of $x_{j}, j \neq i$. It follows from these two facts and from our choice of bases $\mathfrak{B}_{\lambda}, \mathfrak{B}_{0}$ that there exist invertible matrices $E_{\lambda, i}$ of order $n_{i}$ such that

$$
\left[F_{\lambda}^{\prime}\left(a_{\lambda}^{*}\right)\right]_{\mathfrak{B}_{0}}^{\mathfrak{B}_{\lambda}}=E_{\lambda, 1} \oplus \cdots \oplus E_{\lambda, m}
$$

Notice that since $F_{\lambda} \circ F_{-\lambda} \equiv \mathbb{I}$ on a small neighbourhood of $a_{\lambda}^{*}$, we have

$$
\left[F_{-\lambda}^{\prime}\left(a_{\lambda}^{*}\right)\right]_{\mathfrak{B}_{\lambda}}^{\mathfrak{B}_{0}}=E_{\lambda, 1}^{-1} \oplus \cdots \oplus E_{\lambda, m}^{-1} .
$$

The formula for multiplying block matrices together with (4.9), (4.10) and (4.11) proves the result.

\section{The Proof of Theorem 1.2}

This section is devoted to the proofs of Theorem 1.2 and Corollary 1.4. We use tools and techniques developed in Sect. 4 to prove Theorem 1.2 when $A$ is diagonalizable. When $A$ is non-derogatory, our proof of the theorem crucially depends on an explicit construction of a right inverse of the map c passing through the point $A$-which is independent of Sect. 4. But first we prove an important lemma.

Lemma 5.1 Consider $\Omega \subset \mathbb{C}$ and $n \in \mathbb{N}, n \geq 2$, such that $\#(\mathbb{C} \backslash \Omega) \geq 2 n$. Let $A \in S_{n}(\Omega)$ and $\Psi \in \mathcal{O}\left(S_{n}(\Omega), S_{n}(\Omega)\right)$ be such that $\Psi(A)=A$ and $\Psi^{\prime}(A)=\mathbb{I}$. Let $B \in M_{n}(\mathbb{C})$ be such that $B=S A S^{-1}$ for some invertible matrix $S \in M_{n}(\mathbb{C})$. Consider $\Phi \in \mathcal{O}\left(S_{n}(\Omega), M_{n}(\mathbb{C})\right)$ defined by $\Phi=C_{S}{ }^{-1} \circ \Psi \circ C_{S}$, where $C_{S}(W)=$ $S^{-1} W S$ for all $W \in M_{n}(\mathbb{C})$. Then

(1) $\Phi \in \mathcal{O}\left(S_{n}(\Omega), S_{n}(\Omega)\right)$ with $\Phi(B)=B$ and $\Phi^{\prime}(B)=\mathbb{I}$.

(2) Let $G_{\Phi} \in \mathcal{O}\left(\Sigma^{n}(\Omega), \Sigma^{n}(\Omega)\right.$ ) be the map associated to the map $\Phi$ (see Lemma 3.3). Then $G_{\Phi} \equiv G_{\Psi}$.

Proof The proof of Part (1) is straightforward. So let us prove Part (2). Choose a point $x \in \Sigma^{n}(\Omega)$ and fix it. Let $W \in S_{n}(\Omega)$ be such that $\mathbf{c}(W)=x$. Then using the identity $G_{\Phi} \circ \mathbf{c}=\mathbf{c} \circ \Phi$ we see that $G_{\Phi}(x)=\mathbf{c}(\Phi(W))=\mathbf{c}\left(C_{S}{ }^{-1} \circ \Psi \circ C_{S}(W)\right)$. Notice that $\mathbf{c} \circ C_{S}{ }^{-1}=\mathbf{c}$ and hence we have $G_{\Phi}(x)=\mathbf{c}\left(\Psi \circ C_{S}(W)\right)$. Now, we use the identity $G_{\Psi} \circ \mathbf{c}=\mathbf{c} \circ \Psi$, to get $\mathbf{c}\left(\Psi \circ C_{S}(W)\right)=G_{\Psi}\left(\mathbf{c}\left(C_{S}(W)\right)\right.$. Since $\mathbf{c} \circ C_{S}=\mathbf{c}$, we have $G_{\Phi}(x)=G_{\Psi}(\mathbf{c}(W))=G_{\Psi}(x)$. As $x$ is arbitrary, the conclusion follows.

We are now ready for

The proof of Theorem 1.2 When $A$ is diagonalizable Let $A \in S_{n}(\Omega)$ be a diagonalizable matrix and $\Psi \in \mathcal{O}\left(S_{n}(\Omega), S_{n}(\Omega)\right)$ be such that $\Psi(A)=A$ and $\Psi^{\prime}(A)=\mathbb{I}$. 
Because of Lemma 5.1, we can assume, without loss of generality, that $A$ is a diagonal matrix with eigenvalues $\lambda_{i}$ with algebraic multiplicity $n_{i}, i \in[1, \ldots, m]$. We shall now compute the trace of $G_{\Psi}^{\prime}$ at the point $a=\mathbf{c}(A)$. Invoking Lemma 4.2, it is necessary and sufficient to compute the trace of $F_{\Psi}^{\prime}$ at $a^{*}:=\theta(A)$, where $F_{\Psi}$ and $\theta$ are as in Lemma 4.2.

Consider a basis $\mathfrak{B}$ of $\mathbb{C}^{n}$ of the form $[\mathfrak{B}]=\left[\mathfrak{B}_{1}\right] \oplus \cdots \oplus\left[\mathfrak{B}_{m}\right]$, where $\mathfrak{B}_{i}$ is a basis of $\mathbb{C}^{n_{i}}$. Furthermore, for $\mathfrak{B}_{i}:=\left\{\mathbf{v}_{j}^{i}: i \in[1, \ldots, m], j \in\left[1, \ldots, n_{i}\right]\right\}$, let us denote by $\mathbf{V}_{j}^{i} \in \prod_{k=1}^{m} \mathbb{C}^{n_{k}}$ the vector defined by $\mathbf{V}_{j}^{i}:=\left(\mathbf{V}_{j, 1}^{i}, \ldots, \mathbf{V}_{j, k}^{i}, \ldots, \mathbf{V}_{j, m}^{i}\right) \in$ $\prod_{k=1}^{m} \mathbb{C}^{n_{k}}$ such that $\mathbf{V}_{j, k}^{i}=\mathbf{0}$ when $k \neq i$ and $\mathbf{V}_{j, i}^{i}=\mathbf{v}_{j}^{i}$. If we represent $F_{\Psi}=$ $\left(F_{\Psi}^{1}, \ldots, F_{\Psi}^{m}\right)$, we can write $F_{\Psi}^{i}=\sum_{j=1}^{n_{i}} F_{\Psi, j}^{i} \mathbf{v}_{j}^{i}$. Notice that the matrix of the derivative of $F_{\Psi}$ at $a^{*}$ with respect to the basis $\mathfrak{B}$ is

$$
\begin{aligned}
{\left[F_{\Psi}^{\prime}\left(a^{*}\right)\right]_{\mathfrak{B}} } & =\oplus_{i=1}^{m}\left[\frac{\partial F_{\Psi, j}^{i}}{\partial \mathbf{V}_{k}^{i}}\left(a^{*}\right)\right] \text { and } \\
\operatorname{trace}\left[F_{\Psi}^{\prime}\left(a^{*}\right)\right]_{\mathfrak{B}} & =\sum_{i=1}^{m} \operatorname{trace}\left[\frac{\partial F_{\Psi, j}^{i}}{\partial \mathbf{V}_{k}^{i}}\left(a^{*}\right)\right] .
\end{aligned}
$$

Now, we prove the following claim:

Claim The trace of $F_{\Psi}^{\prime}$ at $a^{*}$ with respect to the basis $\mathfrak{B}$ is $n$.

To see the claim, fix $i \in[1, \ldots, m]$. We now consider the map $\Psi_{\lambda_{i}}$ and the associated maps $F_{\Psi_{\lambda_{i}}}$ and $G_{\Psi_{\lambda_{i}}}$ that are obtained by replacing $\lambda$ with $\lambda_{i}$ in the definition of maps $\Psi_{\lambda}, G_{\Psi_{\lambda}}, F_{\Psi_{\lambda}}$ introduced just before Lemma 4.5. Notice that since $A$ is a diagonal matrix so is $A-\lambda_{i} \mathbb{I}$. Moreover, $\sigma\left(A-\lambda_{i} \mathbb{I}\right)=\left\{\mu_{1}, \ldots, \mu_{m}\right\}$ where $\mu_{j}=\lambda_{j}-\lambda_{i}$ for all $j \in[1, \ldots, m]$. In particular $\mu_{i}=0$. We now use Proposition 4.4 by taking $A-\lambda_{i} \mathbb{I}$ as $A, \Psi_{\lambda_{i}}$ as $\Psi$ and $F_{\Psi_{\lambda_{i}}}$ as $F_{\Psi}$. Thus there exists a basis $\mathfrak{D}_{i}$ of $\mathbb{C}^{n_{i}}$ such that with respect to the basis $\mathfrak{D}$ of $\mathbb{C}^{n}$ defined by $[\mathfrak{D}]=\left[\mathfrak{D}_{1}\right] \oplus \cdots \oplus\left[\mathfrak{D}_{m}\right]$, the $i$ th diagonal block of order $n_{i}$ of the matrix $\left[F_{\Psi_{\lambda_{i}}}^{\prime}\left(a_{\lambda_{i}}^{*}\right)\right]_{\mathfrak{D}}$ has trace $n_{i}$. By Lemma 4.5, this latter block is similar to the $i$ th diagonal block of $\left[F_{\Psi}^{\prime}\left(a^{*}\right)\right]_{\mathfrak{B}}$ and hence

$$
\operatorname{trace}\left[\frac{\partial F_{\Psi, j}^{i}}{\partial \mathbf{V}_{k}^{i}}\left(a^{*}\right)\right]=n_{i}
$$

Since the above is true for each $i \in[1, \ldots, m]$, the claim follows.

As mentioned in the first paragraph, the claim implies that the trace of $G_{\Psi}^{\prime}$ at $a$ is equal to $n$. Notice, since $\#(\mathbb{C} \backslash \Omega) \geq 2 n$, by Result 3.1 we know $\Sigma^{n}(\Omega)$ is a Kobayashi complete domain and hence it is taut. So part $(a)$ of Result 2.2 implies that each eigenvalue of $G_{\Psi}^{\prime}(a)$ lies in $\overline{\mathbb{D}}$. These two facts together imply that each eigenvalue of $G_{\Psi}^{\prime}(a)$ is equal to 1 . By Result 2.5 , the sequence $\left\{G_{\Psi}^{k}\right\}$ converges in $\mathcal{O}\left(\Sigma^{n}(\Omega), \Sigma^{n}(\Omega)\right)$ to a holomorphic retraction $\rho: \Sigma^{n}(\Omega) \longrightarrow \Sigma^{n}(\Omega)$. Of course, the holomorphic retract $\rho\left(\Sigma^{n}(\Omega)\right)$, which is a closed submanifold of $\Sigma^{n}(\Omega)$, is fixed point-wise by the map $G$. Now, by Corollary 2.4 , we know that the dimension of the retract is equal to the number of eigenvalues of $G_{\Psi}^{\prime}(a)$ that belong to the boundary of $\mathbb{D}$. Since the latter is equal to $n$, it follows that $\rho\left(\Sigma^{n}(\Omega)\right)=\Sigma^{n}(\Omega)$ whence it follows 
that $G_{\Psi}$ is the identity map on $\Sigma^{n}(\Omega)$. This establishes the conclusion of the theorem when $A$ is diagonalizable.

When $A$ is non-derogatory Since the matrix $A$ is non-derogatory, $A$ is similar to the companion matrix of its characteristic polynomial; see [11, p. 195]. Also, recall the map $\kappa: \Sigma^{n}(\Omega) \longrightarrow S_{n}(\Omega)$, a right inverse of the map c, as in the proof of Lemma 3.3. Using $\kappa$, if $a=\left(a_{1}, \ldots, a_{n}\right)=\mathbf{c}(A)$ then $A$ is non-derogatory if and only if $A$ is similar to

$$
\kappa(a)=\left[\begin{array}{cccc}
0 & & & -a_{k} \\
1 & 0 & & -a_{k-1} \\
& \ddots & \ddots & \vdots \\
0 & & 1 & -a_{1}
\end{array}\right]_{n \times n}
$$

Owing to Lemma 5.1, we can assume that $A=\kappa(a)=\kappa(\mathbf{c}(A))$. Recall that the map $G_{\Psi}$ associated with the map $\Psi$ is given by $G_{\Psi}=\mathbf{c} \circ \Psi \circ \kappa$ (see the proof of Lemma 3.3). Note that $G_{\Psi}(a)=a$ and $G_{\Psi}^{\prime}(a)=\mathbf{c}^{\prime}(\Psi(\kappa(a))) \circ \Psi^{\prime}(\kappa(a)) \circ \kappa^{\prime}(a)$. Since $\Psi(A)=A$ and $\Psi^{\prime}(A)=\mathbb{I}$, we get $G_{\Psi}^{\prime}(a)=\mathbf{c}^{\prime}(A) \circ \kappa^{\prime}(a)$. The map $\kappa$ is a right inverse of c passing through $A$, thus $\mathbf{c}^{\prime}(A) \circ \kappa^{\prime}(a)=\mathbb{I}$ whence it follows that $G_{\Psi}^{\prime}(a)=\mathbb{I}$. We now invoke part $(b)$ of Result 2.2 to conclude that $G_{\Psi}$ is the identity map on $\Sigma^{n}(\Omega)$.

We end this section with the proof of Corollary 1.4 but before that we state a result.

Result 5.2 (Baribeau-Ransford [4]) Let $\mathcal{U}$ be an open subset of $M_{n}(\mathbb{C})$ and let $\Psi$ : $\mathcal{U} \longrightarrow M_{n}(\mathbb{C})$ be a spectrum-preserving $\mathcal{C}^{1}$-diffeomorphism of $\mathcal{U}$ onto $\Psi(\mathcal{U})$. Then $\Psi(W)$ is conjugate to $W$ for any $W \in \mathcal{U}$.

Also, see [5, Théorème 2] for an analytic version of the above result. We now present

The proof of Corollary 1.4 Notice, by Theorem 1.2, $\Psi$ is spectrum-preserving. Since $\Psi^{\prime}(A)=\mathbb{I}$, by the inverse function theorem, there exists a neighbourhood $\mathcal{N}$ of $A$ such that $\Psi: \mathcal{N} \longrightarrow \Psi(\mathcal{N})$ is a biholomorphism. Now, the corollary follows from Result 5.2.

\section{Computation of the Rank of $c^{\prime}(A)$}

In this section, we shall present the proof of Proposition 1.7. As we shall see, a key tool in our proof is the local decomposition of the map $\mathbf{c}$ as described in Lemma 4.1. In what follows, given integers $j<k,[j, \ldots, k]^{2}$ denotes the cartesian product of $[j, \ldots, k]$ with itself. We begin with the case when the matrix $A$ is a nilpotent matrix.

Lemma 6.1 Given $p \in \mathbb{N}, p \geq 2$, let $A \in M_{p}(\mathbb{C})$ be a nilpotent matrix. Then the rank of $\mathbf{c}^{\prime}(A)$ is equal to the degree of the minimal polynomial of $A$. 
Proof Note that for any $S \in M_{p}(\mathbb{C})$ that is invertible, we have $\mathbf{c}^{\prime}(A) H=$ $\mathbf{c}^{\prime}\left(S^{-1} A S\right)\left(S^{-1} H S\right)$. It follows from this that the rank of $\mathbf{c}^{\prime}$ is similarity invariant. Hence we shall assume $A$ to be in a Jordan canonical form. More precisely, we write

$$
A=J_{1}(0) \oplus \cdots \oplus J_{m}(0)
$$

where for each $i \in[1, \ldots, m], J_{i}(0)$ is a Jordan block of size $r_{i}$ with eigenvalue 0 such that $r_{1} \leq r_{2} \leq \cdots \leq r_{m}$. Observe the degree of the minimal polynomial of $A$ is $r_{m}$. For each pair of indices $(j, k) \in[1, \ldots, p]^{2}$, let us denote by $E_{j, k} \in M_{p}(\mathbb{C})$ the matrix whose $(j, k)$ th entry is 1 and every other entry is 0 .

Claim Fix a $(j, k)$ such that $(j, k) \notin\left[r_{i}+1, \ldots, r_{i}+r_{i+1}\right]^{2}$ for every $i \in$ $[0, \ldots, m-1]$, and where $r_{0}:=0$. Then $\mathbf{c}\left(A+\epsilon E_{j, k}\right)=\mathbf{c}(A)$.

To see the claim, first consider the case when $A$ consists of only two Jordan blocks, i.e. $m=2$. In this case, if we change the matrix $A$ to $A+\epsilon E_{j, k}$ with $(j, k)$ as in the claim then the Jordan blocks are unaffected and only one of the cross-diagonal blocks of $A$-which are 0 blocks - gets changed. The claim now easily follows from the following formula for the determinant of a block matrix:

$$
\operatorname{det}\left[\begin{array}{ll}
A & B \\
0 & D
\end{array}\right]=\operatorname{det}(A) \operatorname{det}(D)=\operatorname{det}\left[\begin{array}{ll}
A & 0 \\
C & D
\end{array}\right] .
$$

The general case now follows from the principle of mathematical induction on the number of blocks, together with the formulas above. The upshot of the above claim is that for each $(j, k) \notin\left[r_{i}+1, \ldots, r_{i}+r_{i+1}\right]^{2}$ for every $i \in[0, \ldots, m-1]$, the matrices $E_{j, k}$ belong to the kernel of $\mathbf{c}^{\prime}(A)$.

Now, let $(j, k) \in\left[r_{k_{0}}+1, \ldots, r_{k_{0}}+r_{k_{0}+1}\right]^{2}$ for some fixed $k_{0} \in[0, \ldots, m-1]$. Then the perturbed matrix $A+\epsilon E_{j, k}$ is such that the only block of $A$ that gets changed is the $\left(k_{0}+1\right)$ th Jordan block of size $r_{k_{0}+1}$. Now, by the very definition of $\mathbf{c}$, we have

$$
\operatorname{det}\left(t \mathbb{I}-\left(A+\epsilon E_{j, k}\right)\right)=t^{p}+\sum_{v=1}^{p}(-1)^{v} \mathbf{c}_{v}\left(A+\epsilon E_{j, k}\right) t^{p-v} .
$$

On the other hand, we have

$$
\operatorname{det}\left(t \mathbb{I}-\left(A+\epsilon E_{j, k}\right)\right)=t^{\left(p-r_{k_{0}+1}\right)} \operatorname{det}\left(t \mathbb{I}-J_{k_{0}+1}(\epsilon)\right),
$$

where $J_{k_{0}+1}(\epsilon)$ is obtained from $J_{k_{0}+1}(0)$ by adding $\epsilon$ to its $(j, k)$ th entry and keeping every other entry fixed. Observe that $\operatorname{det}\left(t \mathbb{I}-J_{k_{0}+1}(\epsilon)\right)$ is a monic polynomial of degree $r_{k_{0}+1}$. Hence the coefficient of the term $t^{p-v}$ in $\operatorname{det}\left(t \mathbb{I}-\left(A+\epsilon E_{j, k}\right)\right)$, when $v>r_{k_{0}+1}$, are all 0 . This implies that $\mathbf{c}\left(A+\epsilon E_{j, k}\right)$ is a point in $\mathbb{C}^{p}$, whose $v$ th coordinate is zero for every $v>r_{k_{0}+1}$. Therefore, for each $k_{0} \in[0, \ldots, m-1]$, and for each $(j, k) \in\left[r_{k_{0}}+1, \ldots, r_{k_{0}}+r_{k_{0}+1}\right]^{2}, \mathbf{c}\left(A+\epsilon E_{j, k}\right)$ is a point in $\mathbb{C}^{p}$, all of whose $v$ th coordinates are zero when $v>r_{m}$. This shows that $\operatorname{rank}\left(\mathbf{c}^{\prime}(A)\right) \leq r_{m}$. Next, we shall prove the converse of this inequality.

Consider the matrix $B:=\bigoplus_{i=1}^{m} B_{i}$, where each $B_{i}$ is a companion matrix similar to $J_{i}(0)$. Then $B$ is similar to $A$. Now consider $H \in M_{p}(\mathbb{C})$ and write $H=\left[H_{1}, \ldots, H_{p}\right]$, 
where $H_{i}$ 's are columns of $H$. We choose $H$ in such a way that $H_{i}$ 's are all zero columns when $i \neq p$ and writing $H_{p}=\left(h_{p}, \ldots, h_{1}\right)^{T}$, we have $h_{j}=0$ when $j>r_{m}$. Then we have

$$
\operatorname{det}(t \mathbb{I}-(B+H))=t^{p-r_{m}} \operatorname{det}\left(t \mathbb{I}-\widetilde{B}_{m}\right),
$$

where $\widetilde{B}_{m}$ is a companion matrix of order $r_{m}$ whose last column is the vector $\left(h_{r_{m}}, \ldots, h_{1}\right)^{T}$. Hence we have $\operatorname{det}\left(t \mathbb{I}-\widetilde{B}_{m}\right)=t^{r_{m}}+\sum_{j=1}^{r_{m}}\left(-h_{j}\right) t^{r_{m}-j}$. This, together with the above equation, implies

$$
\operatorname{det}(t \mathbb{I}-(B+H))=t^{p}+\sum_{j=1}^{r_{m}}\left(-h_{j}\right) t^{p-j}
$$

whence $\mathbf{c}(B+H)=\left(h_{1}, \ldots,(-1)^{r_{m}-1} h_{r_{m}}, 0, \ldots, 0\right)$. Now, since $\mathbf{c}(B)=0 \in \mathbb{C}^{p}$, the subspace of $\mathbb{C}^{p},\left\{z \in \mathbb{C}^{p}: z=\left(z_{1}, \ldots, z_{p}\right): z_{j}=0, j>r_{m}\right\}$ is contained in the range space of $\mathbf{c}^{\prime}(B)$. Hence $\operatorname{rank}\left(\mathbf{c}^{\prime}(B)\right)=\operatorname{rank}\left(\mathbf{c}^{\prime}(A)\right) \geq r_{m}$.

The next lemma says that the rank of $\mathbf{c}^{\prime}$ is invariant under translation by a scalar matrix.

Lemma 6.2 Let $A \in M_{n}(\mathbb{C})$ be given and let $\lambda \in \mathbb{C}$ be a fixed complex number. Then the rank of $\mathbf{c}^{\prime}(A)$ is equal to the rank of $\mathbf{c}^{\prime}\left(A_{\lambda}\right)$, where $A_{\lambda}=A-\lambda \mathbb{I}$.

Proof Choose a bounded domain $\Omega$ that contains $\sigma(A)$ and fix it. Recall that $\Omega_{\lambda}:=$ $\{z-\lambda: z \in \Omega\}$. Then $L_{\lambda}\left(S_{n}(\Omega)\right)=S_{n}\left(\Omega_{\lambda}\right)$, where $L_{\lambda}(W)=W-\lambda \mathbb{I}$ for all $W \in M_{n}(\mathbb{C})$. Consider $L_{-\lambda} \in \mathcal{O}\left(S_{n}\left(\Omega_{\lambda}\right), S_{n}(\Omega)\right)$, the inverse of $L_{\lambda}$, which is a biholomorphism that maps $A_{\lambda}$ to $A$. As noted earlier, (see Fig. 3 in Sect. 4.3) there exists a holomorphic map $G_{-\lambda}: \Sigma^{n}\left(\Omega_{\lambda}\right) \longrightarrow \Sigma^{n}(\Omega)$ such that

$$
G_{-\lambda} \circ \mathbf{c}=\mathbf{c} \circ L_{-\lambda}
$$

In fact, $G_{-\lambda}\left(\pi_{n}\left(z_{1}, \ldots, z_{n}\right)\right)=\pi_{n}\left(z_{1}+\lambda, \ldots, z_{n}+\lambda\right)$, where $\pi_{n}: \mathbb{C}^{n} \longrightarrow \mathbb{C}^{n}$ is the symmetrization map. In particular, $G_{-\lambda}$ is a biholomorphism. Now taking the derivatives of both sides in the above equation at $A_{\lambda}$ we get

$$
G_{-\lambda}^{\prime}\left(\mathbf{c}\left(A_{\lambda}\right)\right) \circ \mathbf{c}^{\prime}\left(A_{\lambda}\right)=\mathbf{c}^{\prime}(A) \circ L_{-\lambda}^{\prime}\left(A_{\lambda}\right)
$$

Notice that $L_{-\lambda}^{\prime}\left(A_{\lambda}\right)=\mathbb{I}$ and hence $G_{-\lambda}^{\prime}\left(\mathbf{c}\left(A_{\lambda}\right)\right) \circ \mathbf{c}^{\prime}\left(A_{\lambda}\right)=\mathbf{c}^{\prime}(A)$. Since $G_{-\lambda}^{\prime}\left(\mathbf{c}\left(A_{\lambda}\right)\right)$ is an invertible linear transformation, it follows that $\operatorname{rank}\left(\mathbf{c}^{\prime}\left(A_{\lambda}\right)\right)=\operatorname{rank}\left(\mathbf{c}^{\prime}(A)\right)$.

We are now ready for

The proof of Proposition 1.7 We shall work in the setting of Lemma 4.1. Denote by $\lambda_{i}, i \in[1, \ldots, m]$ the eigenvalues of $A$ having algebraic multiplicity $n_{i}$. Then Lemma 4.1 says that there is a polydisc $P(A ; \delta)$ such that $\mathbf{c}$ decomposes as $\mathbf{c}=\tau \circ \theta$, where $\theta: P(A ; \delta) \longrightarrow \prod_{i=1}^{m} \Sigma^{n_{i}}(\Omega)$ is a holomorphic open map defined by (4.2) 
and $\tau: \prod_{i=1}^{m} \Sigma^{n_{i}}(\Omega) \longrightarrow \Sigma^{n}(\Omega)$ is as defined by (4.1). Moreover, $\tau$ is a biholomorphism from $\theta(P(A ; \delta))$ onto $\mathbf{c}(P(A ; \delta))$. Hence to compute the rank of $\mathbf{c}^{\prime}(A)$ it is sufficient to compute the rank of $\theta^{\prime}(A)$. Let us write $\theta=\left(\theta_{1}, \ldots, \theta_{m}\right)$, where $\theta_{i}: P(A ; \delta) \longrightarrow \Sigma^{n_{i}}(\Omega)$ is a holomorphic map. Observe that, at the tangent space level, we have

$\theta^{\prime}(A): T_{A}(P(A ; \delta)) \equiv \mathbb{C}^{n^{2}} \longrightarrow T_{\theta(A)}\left(\prod_{i=1}^{m} \Sigma^{n_{i}}(\Omega)\right) \equiv \mathbb{C}^{n_{1}} \oplus \mathbb{C}^{n_{2}} \oplus \cdots \oplus \mathbb{C}^{n_{m}}$ and $\theta_{i}^{\prime}(A): T_{A}(P(A ; \delta)) \equiv \mathbb{C}^{n^{2}} \longrightarrow T_{\theta_{i}(A)}\left(\Sigma^{n_{i}}(\Omega)\right) \equiv \mathbb{C}^{n_{i}}$ for every $i \in[1, \ldots, m]$.

As before, we assume $A$ to be in Jordan canonical form and write

$$
A=A_{1} \oplus \cdots \oplus A_{m}, \quad \text { where each } A_{i}=\oplus_{j=1}^{p_{i}} J_{r_{i, j}}\left(\lambda_{i}\right)
$$

Here, $J_{r_{i, j}}\left(\lambda_{i}\right)$ is a Jordan block of size $r_{i, j}$ with eigenvalue $\lambda_{i}$ such that $r_{i, 1} \leq \cdots \leq$ $r_{i, p_{i}}$.

Claim rank $\left(\theta^{\prime}(A)\right)=\sum_{i=1}^{m} \operatorname{rank}\left(\theta_{i}^{\prime}(A)\right)$.

Let us begin with defining certain subspaces of $M_{n}(\mathbb{C})$. Recall that $E_{i, j} \in M_{n}(\mathbb{C})$ is the matrix whose $(i, j)$ th entry is 1 and every other entry is 0 . Now consider

$$
\begin{aligned}
& \mathcal{S}_{0}:=\operatorname{span}\left\{E_{i, j}: \text { changing } A \text { to } A+E_{i, j} \text { does not change any of the blocks } A_{i} \text { for any } i\right\} \\
& \mathcal{S}_{k}:=\operatorname{span}\left\{E_{i, j}: \text { changing } A \text { to } A+E_{i, j} \text { only results in a change in the block } A_{k}\right\}
\end{aligned}
$$

for every $k \in[1, \ldots, m]$. Note that $\mathcal{S}_{l} \perp \mathcal{S}_{k}$ whenever $0 \leq l \neq k \leq m$ and $\mathcal{S}_{0} \oplus \mathcal{S}_{1} \oplus$ $\cdots \oplus \mathcal{S}_{m}=M_{n}(\mathbb{C})=T_{A}(P(A ; \delta))$.

Subclaim For every $i \in[1, \ldots, m], \theta_{i}^{\prime}(A)\left(\mathcal{S}_{i}{ }^{\perp}\right)=0$, i.e. $\theta_{i}^{\prime}(A)(H)=0$ for all $H \in \mathcal{S}_{i}{ }^{\perp}$, which is equivalent to

$$
\lim _{\epsilon \rightarrow 0} \frac{\theta_{i}(A+\epsilon H)-\theta_{i}(A)}{\epsilon}=0 \forall H \in \mathcal{S}_{i}^{\perp}
$$

To prove the subclaim, we begin with noticing that

$$
\mathcal{S}_{i}^{\perp}=\mathcal{S}_{0} \oplus \mathcal{S}_{1} \oplus \cdots \oplus \mathcal{S}_{i-1} \oplus \mathcal{S}_{i+1} \oplus \cdots \oplus \mathcal{S}_{m}
$$

If $H \in \mathcal{S}_{0}$, following the same idea as in the proof of Lemma 6.1, we see that $\theta(A+\epsilon H)=\theta(A)$ for every $\epsilon>0$ that is sufficiently small. This, in particular, implies $\theta_{i}(A+\epsilon H)=\theta_{i}(A)$ for every $H \in \mathcal{S}_{0}$. Hence $\theta_{i}^{\prime}(A)(H)=0$ for all $H \in \mathcal{S}_{0}$.

Now, let $H \in \mathcal{S}_{k}, k \in[1, \ldots, m], k \neq i$. For sufficiently small $\epsilon>0, A+\epsilon H \in$ $P(A ; \delta)$. Further only the $k$ th diagonal block of $A$ is perturbed when we change $A$ to $A+\epsilon H$. This implies the value of $\theta_{i}$ under such perturbation is unaffected, i.e. $\theta_{i}(A+\epsilon H)=\theta_{i}(A)$ for all $H \in \mathcal{S}_{k}, k \in[1, \ldots, m], k \neq i$. Hence $\mathcal{S}_{k} \subset \operatorname{Ker}\left(\theta_{i}^{\prime}(A)\right)$ 
for every $k \in[0, \ldots, m], k \neq i$. Since $\operatorname{Ker}\left(\theta_{i}^{\prime}(A)\right)$ is a subspace of $M_{n}(\mathbb{C})$, it follows that $\mathcal{S}_{i}^{\perp} \subset \operatorname{Ker}\left(\theta_{i}^{\prime}(A)\right)$ for every $i \in[1, \ldots, m]$. Hence

$$
\left[\theta_{i}^{\prime}(A)\right]_{n_{i} \times n^{2}}=\left([0]_{n_{i} \times n_{0}}[0]_{n_{i} \times n_{1}{ }^{2}} \ldots\left[\left.\theta_{i}^{\prime}(A)\right|_{\mathcal{S}_{i}}\right]_{n_{i} \times n_{i}{ }^{2}} \ldots[0]_{n_{i} \times n_{m}{ }^{2}}\right)
$$

Here $n_{0}$ is the dimension of the subspace $V_{0}$. So from the form of the matrix

$$
\left[\theta^{\prime}(A)\right]_{n \times n^{2}}=\left(\begin{array}{c}
{\left[\theta_{1}^{\prime}(A)\right]} \\
{\left[\theta_{2}^{\prime}(A)\right]} \\
\vdots \\
{\left[\theta_{m}^{\prime}(A)\right]}
\end{array}\right),
$$

we see that the non-zero block of matrices $\left[\theta_{i}^{\prime}(A)\right]_{n_{i} \times n^{2}}$ shifts rightward when $i$ increases from 1 to $m$. This, in particular, implies that the rank of the matrix $\left[\theta^{\prime}(A)\right]_{n \times n^{2}}$ is equal to the sum of the rank of the matrices $\left[\left.\theta_{i}^{\prime}(A)\right|_{\mathcal{S}_{i}}\right]_{n_{i} \times n_{i}{ }^{2}}$ whence our claim follows.

Now fix an $i \in[1, \ldots, m]$, we shall now show that $\operatorname{rank}\left(\theta_{i}^{\prime}(A)\right)=r_{i, p_{i}}$. Because of Lemma 6.2, we can assume without loss of generality that $\lambda_{i}=0$. Moreover, from the above discussion, it is clear that

$$
\theta_{i}^{\prime}(A)\left(M_{n}(\mathbb{C})\right)=\theta_{i}^{\prime}\left(\mathcal{S}_{i} \oplus \mathcal{S}_{i}^{\perp}\right)=\theta_{i}^{\prime}\left(\mathcal{S}_{i}\right)
$$

Notice that if we take a matrix $H \in \mathcal{S}_{i}$ and decompose it in blocks corresponding to the blocks of $A$ as above then the only non-zero block is the $i$ th diagonal block of size $n_{i}$. With this observation in hand, proceeding exactly as in the proof of Lemma 6.1, we see that the rank of $\theta_{i}^{\prime}(A)=r_{i}, p_{i}$. Since the choice of $i$ was arbitrary, using the claim above, we get that the rank of $\theta^{\prime}(A)=\sum_{i=1}^{m} r_{i, p_{i}}=$ degree of the minimal polynomial of $A$.

See [15] for a simple proof of Proposition 1.7 when $A$ is a non-derogatory matrix.

\section{The Proof of Theorem 1.8 and the Case of $3 \times 3$ Matrices}

We present the proof of Theorem 1.8 in this section. As mentioned in the introduction, a key result in the proof of this theorem is Proposition 1.7. The other tool is a result due to Vigué stated in Sect. 2.

The proof of Theorem 1.8 We consider the map $G_{\Psi}$ associated to the map $\Psi$ as in Lemma 3.3. The map $G_{\Psi}$ satisfies $G_{\Psi} \circ \mathbf{c}=\mathbf{c} \circ \Psi$. Since $\Psi(A)=A$, we see that $G_{\Psi}(\mathbf{c}(A))=\mathbf{c}(A)$. Write $a=\mathbf{c}(A)$. Then, by Result 2.6, the fixed-point set of the map $G_{\Psi}$ denoted by $\operatorname{Fix}\left(G_{\Psi}\right)$ is a closed complex submanifold such that

$$
T_{a}\left(\operatorname{Fix}\left(G_{\Psi}\right)\right)=\left\{\xi \in T_{a}\left(\Sigma^{n}(\Omega)\right): G_{\Psi}^{\prime}(a) \xi=\xi\right\} .
$$

Differentiating both sides of $G_{\Psi} \circ \mathbf{c}=\mathbf{c} \circ \Psi$ at $A$ gives $G_{\Psi}^{\prime}(a) \circ \mathbf{c}^{\prime}(A)=\mathbf{c}^{\prime}(\Psi(A)) \circ$ $\Psi^{\prime}(A)$. Substituting $\Psi(A)=A$ and $\Psi^{\prime}(A)=\mathbb{I}$, we get $G_{\Psi}^{\prime}(a) \circ \mathbf{c}^{\prime}(A)=\mathbf{c}^{\prime}(A)$. 
In particular, every $\xi \in T_{a}\left(\Sigma^{n}(\Omega)\right)$ that belongs to the range space of $\mathbf{c}^{\prime}(A)$ is in $T_{a}\left(\operatorname{Fix}\left(G_{\Psi}\right)\right)$. Hence

$$
\operatorname{dim}_{\mathbb{C}}\left[T_{a}\left(\operatorname{Fix}\left(G_{\Psi}\right)\right)\right] \geq \operatorname{rank}\left(\mathbf{c}^{\prime}(A)\right)
$$

By Proposition 1.7, the rank of $\mathbf{c}^{\prime}(A)$ is equal to the degree of the minimal polynomial of $A$. This implies that the dimension of the fixed-point set Fix $\left(G_{\Psi}\right)$ is greater than or equal to the degree of the minimal polynomial of $A$. Clearly, if $W \in S_{n}(\Omega)$ be such that $\mathbf{c}(W) \in \operatorname{Fix}\left(G_{\Psi}\right)$ then $\mathbf{c}(W)=\mathbf{c}(\Psi(W))$. Taking $\mathscr{S}=\operatorname{Fix}\left(G_{\Psi}\right)$, Theorem 1.8 follows.

\section{The Case When $\boldsymbol{A}$ is a $\mathbf{3} \times \mathbf{3}$ Matrix}

Let $\Omega \subset \mathbb{C}$ be a domain such that $\#(\mathbb{C} \backslash \Omega) \geq 6$. Consider $\Psi \in \mathcal{O}\left(S_{3}(\Omega), S_{3}(\Omega)\right)$ such that $\Psi(A)=A$ and $\Psi^{\prime}(A)=\mathbb{I}$. To analyse this case completely, we present a lemma. For this, let $\Omega, A, \Psi$ be as in the statement of Theorem 1.2. Choose a $\lambda \in \mathbb{C}$ and consider $\Omega_{\lambda}=\{z-\lambda: z \in \Omega\}$ as defined before. Note, if $\Omega$ satisfies the cardinality condition then so does $\Omega_{\lambda}$. Now recall $\Psi_{\lambda} \in \mathcal{O}\left(S_{n}\left(\Omega_{\lambda}\right), S_{n}\left(\Omega_{\lambda}\right)\right)$ defined by

$$
\Psi_{\lambda}=L_{\lambda} \circ \Psi \circ L_{-\lambda}
$$

where $L_{\lambda}(W):=W-\lambda \mathbb{I}$ and $L_{-\lambda}$ is the inverse of $L_{\lambda}$. Note, $\Psi_{\lambda}\left(A_{\lambda}\right)=A_{\lambda}$ and $\Psi^{\prime}\left(A_{\lambda}\right)=\mathbb{I}$, where $A_{\lambda}:=A-\lambda \mathbb{I}$.

Lemma 7.1 Let $G_{\Psi_{\lambda}} \in \mathcal{O}\left(\Sigma^{n}\left(\Omega_{\lambda}\right)\right.$, $\left.\Sigma^{n}\left(\Omega_{\lambda}\right)\right)$ be the map associated to the map $\Psi_{\lambda}$ as given by Lemma 3.3. Then $G_{\Psi_{\lambda}}$ is the identity map on $\Sigma^{n}\left(\Omega_{\lambda}\right)$ if and only if $G_{\Psi}$ is the identity map on $\Sigma^{n}(\Omega)$.

Proof It is easy to see that $G_{\Psi_{\lambda}}=G_{\lambda} \circ G_{\Psi} \circ G_{-\lambda}$, where $G_{-\lambda}\left(\pi_{n}\left(z_{1}, \ldots, z_{n}\right)\right)=$ $\pi_{n}\left(z_{1}+\lambda, \ldots, z_{n}+\lambda\right)$, where $\pi_{n}: \mathbb{C}^{n} \longrightarrow \mathbb{C}^{n}$ is the symmetrization map. As noted before, $G_{-\lambda}$ is a biholomorphism with the inverse $G_{\lambda}$ defined by $G_{\lambda}\left(\pi_{n}\left(z_{1}, \ldots, z_{n}\right)\right)=\pi_{n}\left(z_{1}-\lambda, \ldots, z_{n}-\lambda\right)$. Thus the lemma follows.

We now analyse the case of order 3 matrices. First, using Lemma 5.1, we shall assume $A$ to be in the Jordan canonical form. Also, applying Lemma 7.1, without loss of generality, we can assume that $0 \in \sigma(A)$. Moreover, if we let

$$
n_{A}(\lambda):=\text { the number of Jordan blocks corresponding to the eigenvalue } \lambda
$$

then we can assume that $n_{A}(0) \geq n_{A}(\lambda)$, where $\lambda \in \sigma(A)$ and $\lambda \neq 0$. Notice that in the case when $1=n_{A}(0) \geq n_{A}(\lambda), A$ is non-derogatory and we know that $\Psi$ is spectrum-preserving by Theorem 1.2. In the case when $n_{A}(0)=n, A$ is the zero matrix and in this case too we are done by Theorem 1.2. In the case $n=3$, we are only left to the case $n_{A}(0)=2$. Let $r_{1}, r_{2}$ be the sizes of these two blocks with $r_{1} \leq r_{2}$. The only choices that we are left with are following: 
(a) The case $r_{1}=r_{2}=1$. Then

$$
A=\left(\begin{array}{lll}
0 & 0 & 0 \\
0 & 0 & 0 \\
0 & 0 & \lambda
\end{array}\right) \text { and } \lambda \neq 0 .
$$

Since $A$ is diagonal, it follows from Theorem 1.2 that $\Psi$ is spectrum-preserving. (b) The case $r_{1}=1, r_{2}=2$. Then

$$
A=\left(\begin{array}{lll}
0 & 0 & 0 \\
0 & 0 & 1 \\
0 & 0 & 0
\end{array}\right)
$$

Notice that $A$ is a nilpotent matrix of order 2. The degree of the minimal polynomial for $A$ is 2 . In this case, by Theorem 1.8 , we get that $G_{\Psi}$ is the identity map on a closed complex submanifold of $\Sigma^{n}(\Omega)$ of complex dimension at least 2 . We are not able to obtain any further information about $G_{\Psi}$ in this case.

\section{Appendix: $\mathrm{c}$ is an Open Map}

In this section, we prove that the map $\mathbf{c}$ that appears in the article is an open map. This is Proposition 7.3 below. Not only this could be of independent interest to the reader but also in Sect. 4.1, we refer to the proof of this proposition to conclude that the map $\theta$ is an open map. Before we present our proof, we need the following result.

Result 7.2 Let $p_{1}, p_{2}$ be two polynomials of degree $n$ of the form

$$
\begin{aligned}
& p_{1}(t)=a_{n}+a_{n-1} t+\cdots+a_{1} t^{n-1}+t^{n}, \\
& p_{2}(t)=b_{n}+b_{n-1} t+\cdots+b_{1} t^{n-1}+t^{n} .
\end{aligned}
$$

Let $T:=\max \left\{1,\left|a_{1}\right|,\left|b_{1}\right|,\left|a_{2}\right|^{1 / 2},\left|b_{2}\right|^{1 / 2}, \ldots,\left|a_{n}\right|^{1 / n},\left|b_{n}\right|^{1 / n}\right\}$. If $\alpha_{j}, j \in[1, \ldots, n]$ are the roots of $p_{1}$ then there is an ordering of the roots of $p_{2}, \beta_{1}, \beta_{2}, \ldots, \beta_{n}$, such that

$$
\left|\beta_{j}-\alpha_{j}\right| \leq 4 n T\left(\|a-b\|_{2}\right)^{\frac{1}{n}},
$$

where $\|a-b\|_{2}:=\left(\sum_{j=1}^{n}\left|a_{j}-b_{j}\right|^{2}\right)^{1 / 2}$.

The above result is due to A. M. Ostrowski; see, for instance [6]. Now, we prove

Proposition 7.3 The map c : $M_{n}(\mathbb{C}) \longrightarrow \mathbb{C}^{n}$ defined by $\mathbf{c}(B):=b=\left(b_{1}, \ldots, b_{n}\right)$, where $b$ is such that the polynomial $t^{n}+\sum_{k=1}^{n}(-1)^{k} b_{k} t^{n-k}$ is the characteristic polynomial of $B$, is an open map. 
Proof Let $\mathcal{U} \subseteq M_{n}(\mathbb{C})$ be a non-empty open set. Consider $\mathbf{c}(\mathcal{U})$ and let $x \in \mathbf{c}(\mathcal{U})$ be a fixed point. We shall show that there exists an $\epsilon>0$ such that $\mathbb{B}(x, \epsilon):=\left\{y \in \mathbb{C}^{n}\right.$ : $\left.\|x-y\|_{2}<\epsilon\right\} \subset \mathbf{c}(\mathcal{U})$. Choose $X \in \mathcal{U}$ such that $\mathbf{c}(X)=x$ and write

$$
X=S\left(\operatorname{diag}\left(\lambda_{1}, \ldots, \lambda_{n}\right)+U\right) S^{-1}
$$

where $\lambda_{i}$ 's are eigenvalues of $X, S \in M_{n}(\mathbb{C})$ is an invertible matrix and $U$ is a strictly upper triangular matrix.

Fix an $r \in(0,1)$ and consider $\mathbb{B}(x, r)$. Then for any $y \in \mathbb{B}(x, r)$, by Result 7.2, there exist complex numbers $\mu_{1}, \ldots, \mu_{n}$-that are roots of the polynomial $t^{n}+\sum_{k=1}^{n}(-1)^{k} y_{k} t^{n-k}-$ such that

$$
\left|\lambda_{j}-\mu_{j}\right| \leq 4 n T r^{1 / n}
$$

where $T=\max \left\{1,\left|y_{1}\right|,\left|x_{1}\right|,\left|y_{2}\right|^{1 / 2},\left|x_{2}\right|^{1 / 2}, \ldots,\left|y_{n}\right|^{1 / n},\left|x_{n}\right|^{1 / n}\right\}$ and $x_{j}, y_{j}, j \in$ $[1, \ldots, m]$, are the coordinates of $x$ and $y$, respectively. Since $\left|y_{j}-x_{j}\right| \leq r<1$, we have $\left|y_{j}\right| \leq\left|x_{j}\right|+1$ for every $j \in[1, \ldots, m]$. This shows that the constant $T$ could be chosen in a way so that it only depends on $x$ and $n$.

Now consider $Y:=S\left(\operatorname{diag}\left(\mu_{1}, \ldots, \mu_{n}\right)+U\right) S^{-1}$, where $S$ and $U$ are as in (7.1). Then $Y-X=S\left(\operatorname{diag}\left(\mu_{1}-\lambda_{1}, \ldots, \mu_{n}-\lambda_{n}\right)\right) S^{-1}$. Hence

$$
\begin{aligned}
\|Y-X\|_{\text {op }} & \leq\|S\|_{\text {op }}\left\|S^{-1}\right\|_{\text {op }} \max \left\{\left|\mu_{j}-\lambda_{j}\right|: j \in[1, \ldots, n]\right\} \\
& \leq\|S\|_{\text {op }}\left\|S^{-1}\right\|_{\text {op }} 4 n T r^{1 / n} .
\end{aligned}
$$

Since $X \in \mathcal{U}$ and $\mathcal{U}$ is an open set, there exists a $\delta>0$ such that the set $\left\{W \in M_{n}(\mathbb{C})\right.$ : $\left.\|W-X\|_{\text {op }}<\delta\right\} \subset \mathcal{U}$. Now, we choose an $r$ such that $\|S\|_{\text {op }}\left\|S^{-1}\right\|_{\text {op }} 4 n T r^{1 / n}<\delta$. Then $Y \in\left\{W \in M_{n}(\mathbb{C}):\|W-X\|_{\text {op }}<\delta\right\} \subset \mathcal{U}$. Note, $\mathbf{c}(Y)=y \in \mathbb{B}(x, \epsilon)$, where $\epsilon=r$. This proves that for any arbitrary $x \in \mathbf{c}(\mathcal{U})$, there exists an $\epsilon>0$-depending only on $x$ and $n$-such that $\mathbb{B}(x, \epsilon) \subset \mathbf{c}(\mathcal{U})$. Hence $\mathbf{c}(\mathcal{U})$ is an open set.

Acknowledgements The second author is supported by a postdoctoral fellowship from the Harish-Chandra Research Institute (HBNI), Prayagraj. The third author is supported by a scholarship from the National Board for Higher Mathematics (Ref. No. 2/39(2)/2016/NBHM/R\&D-II/11411).

\section{References}

1. Abate, M.: Iterates and semigroups on taut manifolds. Giornate di Geometria Analitica e Analisi Complessa, pp. 3-13 (1988)

2. Abate, M.: Iteration Theory of Holomorphic Maps on Taut Manifolds. Mediterranean Press, Cosenza (1989)

3. Andrist, R.B., Kutzschebauch, F.: The fibred density property and the automorphism group of the spectral ball. Math. Ann. 370(1-2), 917-936 (2018)

4. Baribeau, L., Ransford, T.: Non-linear spectrum-preserving maps. Bull. Lond. Math. Soc. 32(1), 8-14 (2000)

5. Baribeau, L., Roy, S.: Caractérisation spectrale de la forme de Jordan. Linear Algebra Appl. 320(1-3), 183-191 (2000) 
6. Beauzamy, B.: How the roots of a polynomial vary with its coefficients: a local quantitative result. Canad. Math. Bull. 42(1), 3-12 (1999)

7. Bharali, G.: Some new observations on interpolation in the spectral unit ball. Integr. Equ. Oper. Theory 59(3), 329-343 (2007)

8. Bhatia, R.: Perturbation Bounds for Matrix Eigenvalues. Classics in Applied Mathematics. Society for Industrial and Applied Mathematics, Philadelphia (2007). https://doi.org/10.1137/1.9780898719079

9. Chandel, V.S.: Certain non-homogeneous matricial domains and Pick-Nevanlinna interpolation problem. Internat. J. Math. 32 (11), 2150083 (2021)

10. Costara, C., Ransford, T.: On local irreducibility of the spectrum. Proc. Am. Math. Soc. 135(9), 27792784 (2007)

11. Horn, R.A., Johnson, C.R.: Matrix Analysis, 2nd edn. Cambridge University Press, Cambridge (2013)

12. Jarnicki, M., Pflug, P.: Invariant Distances and Metrics in Complex Analysis. 2nd extended ed. Walter de Gruyter, Berlin (2013)

13. Kobayashi, S.: Hyperbolic Manifolds and Holomorphic Mappings. Pure and Applied Mathematics. Marcel Dekker Inc, New York (1970)

14. Kobayashi, S.: Hyperbolic Complex Spaces, Grundlehren der Mathematischen Wissenschaften, vol. 318. Springer, Berlin (1998)

15. Nikolov, N., Thomas, P.J., Zwonek, W.: Discontinuity of the Lempert function and the KobayashiRoyden metric of the spectral ball. Integr. Equ. Oper. Theory 61, 401-412 (2008)

16. Nikolov, N., Pflug, P., Thomas, P.J.: Spectral Nevanlinna-Pick and Carathéodory-Fejér problems for $n \leq 3$. Indiana Univ. Math. J. 60(3), 883-893 (2011)

17. Ransford, T.J., White, M.C.: Holomorphic self-maps of the spectral unit ball. Bull. Lond. Math. Soc. 23(3), 256-262 (1991)

18. Song, Y.: A note on the variation of the spectrum of an arbitrary matrix. Linear Algebra Appl. 342, 41-46 (2002)

19. Sun, Ji.-guang: On the variation of the spectrum of a normal matrix. Linear Algebra Appl. 246, 215-223 (1996)

20. Vigué, J.-P.: Points fixes d'une limite d'applications holomorphes. Bull. Sci. Math. (2) 110(4), 411-424 (1986)

21. Wu, H.: Normal families of holomorphic mappings. Acta Math. 119, 193-233 (1967)

22. Zwonek, W.: Proper holomorphic mappings of the spectral unit ball. Proc. Am. Math. Soc. 136(8), 2869-2874 (2008)

23. Zwonek, W.: Function theoretic properties of symmetric powers of Complex Manifolds. J. Geom. Anal. 30, 1226-1237 (2020)

Publisher's Note Springer Nature remains neutral with regard to jurisdictional claims in published maps and institutional affiliations. 